\title{
A Monte Carlo method with negative particles for Coulomb collisions*
}

\author{
Bokai Yan ${ }^{\dagger}$ and Russel E. Caflisch ${ }^{\dagger}$
}

June 8, 2015

\begin{abstract}
In this work we propose a novel negative particle method for the general bilinear collision operators in the spatial homogeneous case and apply it to Coulomb collisions. This new method successfully reduces the growth of particle numbers from the numerical time scale to the physical time scale for Coulomb collisions. We also propose a particle resampling method which reduces the particle number to further improve the efficiency. Various numerical simulations are performed to demonstrate the accuracy and efficiency of the method.
\end{abstract}

Keywords. Coulomb Collisions, Monte Carlo Method, Negative Particle Method, PredictionCorrection, Boltzmann equation.

\section{Introduction}

In the numerical study of non-equilibrium plasma physics, the simulation of long range Coulomb collisions between charged particles (electrons and ions) is of crucial importance. The particles are represented by a distribution $f(t, \mathbf{v})$ in phase space, at time $t$ with velocity $\mathbf{v}$. Coulomb collisions can be modeled by a Landau-Fokker-Planck (LFP) equation as the grazing limit of the Boltzmann equation. Direct Simulation Monte Carlo (DSMC) [4] is the prevalent numerical method for solving LFP equations, since deterministic methods suffer from formidable computational costs due to the high dimensionality of the distribution function.

Two widely used DSMC methods are those of by Takizuka-Abe [21] (henceforth TA) and Nanbu [14], and recently studied extensively in, for example, [9, 10, 22]. Bobylev and Nanbu [5] derived a general formulation for the approximation of LFP equations. However these (and all DSMC) methods become inefficient as the distribution approaches equilibrium, since most computation is spent on the collisions between particles sampled from the equilibrium part. A hybrid method is more favorable in this case, by evolving the equilibrium part according to a fluid equation and sampling particles from only the remaining part. Caflisch et al. [6] introduced thermalization/dethermalization (TD) methods by splitting the distribution into

$$
f(\mathbf{v})=m(\mathbf{v})+f_{p}(\mathbf{v}),
$$

i.e. an equilibrium part $m$ and a positive deviation part $f_{p}(\mathbf{v}) \geq 0$. After performing collisions in each step, a thermalization/dethermalization step is applied as a reorganization of the splitting (1.1). Later Ricketson et al. [17] improved this method by associating each numerical particle with

\footnotetext{
*This research was supported by DOE DE-FG02-13ER26152/DE-SC0010613.

${ }^{\dagger}$ Mathematics Department, University of California at Los Angeles, Los Angeles, CA 90095-1555 USA. byan@math.ucla.edu, caflisch@math.ucla.edu
} 
an entropy which provides more accurate information on whether thermalization/dethermalization is needed. Another hybrid method is proposed in [20].

The splitting (1.1) is less efficient if defects exist in the equilibrium $m$, since one needs to take the equilibrium below the whole distribution (i.e. $m \leq f)$, leaving the large remaining part $(f-m)$ to be represented by particles. In this work we use "negative" particles, which represent the defects in equilibrium. More specifically, we write

$$
f(\mathbf{v})=m(\mathbf{v})+f_{p}(\mathbf{v})-f_{n}(\mathbf{v}),
$$

with $f_{p}(\mathbf{v}) \geq 0$ and $f_{n}(\mathbf{v}) \geq 0$ the positive and negative parts of the deviation from equilibrium $m(\mathbf{v})$. Positive and negative particles are sampled from $f_{p}$ and $f_{n}$ respectively. Hadjiconstantinou and co-workers $[2,3,11]$ studied the collisions involving negative particles in rarefied gas and developed the low-variance deviational simulation Monte Carlo (LVDSMC) method. Our work, described below, is the first use of negatives particles in a DSMC method for Coulomb collisions.

The major problem in a negative particle method is that the total number of positive and negative particles increases due to collisions involving a negative particle. In the DSMC method for rarefied gas, only a number of $\mathcal{O}(\Delta t)$ collisions are performed in one step, which makes $N(t)$, the total number of particles, grow exponentially on a physical time scale. To address this growth, [2] constructed a mesh with small grid size in phase space, and in each grid cell the particles with opposite signs are removed in pairs. Later a gridless method was developed for linearized variable hard sphere (VHS) type collisions in [12]. Besides, weighted particles have also been studied $([8,1])$ in rarefied gas collisions for variance reduction. We refer the reader to a recent review [16] for more results.

However, a direct application of the negative particle method on Coulomb collisions leads to a much more severe problem in the growth of the number of particles. In the TA method and Nanbu's method for Coulomb collisions, every particle collides in every time step, which (more than) doubles $N(t)$ in every step. As a result the particle number grows in the numerical scale; i.e., $N(t)$ grows unbounded as time step $\Delta t \rightarrow 0$. We give more details in Section 2.2.

This work contains three main contributions.

- We propose a novel negative particle method for general bilinear collisions (including both RGD and Coulomb collisions), in which the particle number only grows in the physical time scale, i.e., independent of time step $\Delta t$. Two key ideas are:

- We propose a new decomposition formulation for the collision operator. In each time step, the positive/negative particles undergo two processes: regular collisions with particles sampled from $f$; and particles sampled from a source term of size $\mathcal{O}(\Delta t)$. Hence only extra $\mathcal{O}(\Delta t)$ particles are created.

- We propose to use a small number of independently evolved particles (called "F-particles" below) to represent the particles sampled from $f$ in the first process.

This is similar to a prediction-correction type method. The small number of independently evolved particles give a coarse solution to $f$. Then a finer solution is obtained by evolving the positive and negative particles. The applicability of this method is not restricted to the Coulomb collisions, but for the general bilinear operators. To the best knowledge of the authors these ideas have not been used before.

- We propose a particle resampling method to reduce the number of particles when it grows over some threshold.

- We apply this new method to Coulomb collisions. This method require sampling of particles from the source term $Q\left(f_{p}-f_{n}, m\right)$, the change in the Maxwellian component $m$ due to 
collisions with positive and negative particles. We perform a detailed analysis of this source term and design an efficient sampling method when $Q$ is the LFP operator.

These techniques lead to a new negative particle method which is much more accurate and efficient than the existing methods for Coulomb collisions. In this work we focus on the spatially homogeneous case. The extension to spatially inhomogeneous simulation deserves further investigation.

The remainder of this paper is organized as follows. In Section 2 the negative particle method for rarefied gas collisions is reviewed and then generalized to Coulomb collisions. We show that this method is not stable for Coulomb collisions due to the severe growth of the number of particles. Next in Section 3 we describe a new negative particle method for general binary collisions. A new particle reduction technique is also introduced in Section 3.5. Then in Section 4 we apply this method to the LFP equation, with details on how to sample from the source term. The whole algorithm is summarized in Section 5, with some discussions on acceleration techniques. Finally we give some numerical results in Section 6 to illustrate the high accuracy and efficiency. Conclusions are included in Section 7. Various details of the analysis are provided in four appendices.

\section{Negative particle methods}

In a negative particle method, the distribution $f$ is split into

$$
f(\mathbf{v}, t)=m(\mathbf{v}, t)+f_{d}(\mathbf{v}, t)=m(\mathbf{v}, t)+f_{p}(\mathbf{v}, t)-f_{n}(\mathbf{v}, t),
$$

where $m$ is a Maxwellian distribution which does not necessary have the same moments as $f . f_{d}(\mathbf{v})$ is the deviational distribution which might be negative for some $\mathbf{v} . f_{p}$ and $f_{n}$ are the positive and negative parts of $f_{d}$.

Denote the densities of each part as

$$
\rho=\int f(\mathbf{v}, t) \mathrm{d} \mathbf{v}, \quad \rho_{m}=\int m(\mathbf{v}, t) \mathrm{d} \mathbf{v}, \quad \rho_{p}=\int f_{p}(\mathbf{v}, t) \mathrm{d} \mathbf{v}, \quad \rho_{n}=\int f_{n}(\mathbf{v}, t) \mathrm{d} \mathbf{v},
$$

and the normalized distributions

$$
\hat{f}=\frac{f}{\rho}, \quad \hat{m}=\frac{m}{\rho_{m}}, \quad \hat{f}_{p}=\frac{f_{p}}{\rho_{p}}, \quad \hat{f}_{n}=\frac{f_{n}}{\rho_{n}} .
$$

The positive part $f_{p}(\mathbf{v}, t) \geq 0$ and the negative part $f_{n}(\mathbf{v}, t) \geq 0$ are represented by particles, called P-particles and N-particles respectively, whose numbers are denoted by $N_{p}$ and $N_{n}$. With the effective number $N_{\text {eff }}=\frac{\# \text { real particles }}{\# \text { numerical particles }}$, one has

$$
N_{p}=\frac{\rho_{p}}{N_{\mathrm{eff}}}, \quad N_{n}=\frac{\rho_{n}}{N_{\mathrm{eff}}} .
$$

The Maxwellian part $m(\mathbf{v}, t)$ is represented by its moments. Denote $N_{m}=\frac{\rho_{m}}{N_{\text {eff }}}$ as the number of M-particles. M-particles are not tracked and only generated from $m$ whenever needed. $m$ is chosen to be an (approximately) fixed Maxwellian, for simplicity.

We call the collision between an A-particle and a B-particle to be an A-B collision, with $A, B \in$ $\{P, N, M\}$.

\subsection{Negative particle methods for rarefied gas}

A collision algorithm in rarefied gas with negative particles was developed in $[2,3]$. In this section we review this algorithm using a slightly different formulation. 
Binary collisions in rarefied gas can be described by the spatially homogeneous Boltzmann equation,

$$
\frac{\mathrm{d} f}{\mathrm{~d} t}=Q(f, f)
$$

with the bilinear collision operator

$$
\begin{aligned}
Q(f, g)(\mathbf{v}) & =\iint_{\mathbb{R}^{3} \times \mathbb{S}^{2}} B\left(\left|\mathbf{v}-\mathbf{v}_{*}\right|, \cos \theta\right)\left(f_{*}^{\prime} g^{\prime}-f_{*} g\right) \mathrm{d} \mathbf{v}_{*} \mathrm{~d} \sigma \\
& =Q^{+}(f, g)-Q^{-}(f) g .
\end{aligned}
$$

in which $Q^{+}$and $Q^{-}$are positive. We refer the reader to [7] for a detailed description of the Boltzmann operator.

Applying the representation (2.1), equation (2.2) can be expanded as

$$
\begin{aligned}
\frac{\mathrm{d} f}{\mathrm{~d} t}= & \left(Q^{+}\left(f_{p}, f_{p}\right)-Q^{-}\left(f_{p}\right) f_{p}\right) \\
& +\left(-Q^{+}\left(f_{n}, f_{p}\right)-Q^{+}\left(f_{p}, f_{n}\right)+Q^{-}\left(f_{n}\right) f_{p}+Q^{-}\left(f_{p}\right) f_{n}\right) \\
& +\left(Q^{+}\left(f_{n}, f_{n}\right)-Q^{-}\left(f_{n}\right) f_{n}\right) \\
& +\left(Q^{+}\left(f_{p}, m\right)+Q^{+}\left(m, f_{p}\right)-Q^{-}\left(f_{p}\right) m-Q^{-}(m) f_{p}\right) \\
& +\left(-Q^{+}\left(f_{n}, m\right)-Q^{+}\left(m, f_{n}\right)+Q^{-}\left(f_{n}\right) m+Q^{-}(m) f_{n}\right),
\end{aligned}
$$

representing P-P, P-N, N-N, P-M and N-M collisions respectively in each line. Here the $Q(m, m)$ term is dropped since it is always 0. Decompose (2.4) into three equations,

$$
\begin{aligned}
& \frac{\mathrm{d} m}{\mathrm{~d} t}=0, \\
& \begin{aligned}
\frac{\mathrm{d} f_{p}}{\mathrm{~d} t}= & \left(Q^{+}\left(m, f_{p}\right)+Q^{+}\left(f_{p}, m\right)+Q^{+}\left(f_{p}, f_{p}\right)+Q^{+}\left(f_{n}, f_{n}\right)\right) \\
& -\left(Q^{-}(m)+Q^{-}\left(f_{p}\right)-Q^{-}\left(f_{n}\right)\right) f_{p}+Q^{-}\left(f_{n}\right) m
\end{aligned} \\
& \begin{aligned}
\frac{\mathrm{d} f_{n}}{\mathrm{~d} t}= & \left(Q^{+}\left(m, f_{n}\right)+Q^{+}\left(f_{n}, m\right)+Q^{+}\left(f_{p}, f_{n}\right)+Q^{+}\left(f_{n}, f_{p}\right)\right) \\
& \quad-\left(Q^{-}(m)+Q^{-}\left(f_{p}\right)-Q^{-}\left(f_{n}\right)\right) f_{n}+Q^{-}\left(f_{p}\right) m
\end{aligned}
\end{aligned}
$$

Here the Maxwellian part is kept invariant. In the equation for $f_{p}$, we list all the positive gain terms, the positive term $Q^{-}\left(f_{n}\right) m$ and the loss terms in the form of $Q^{-}(\cdot) f_{p}$. The remaining terms are left in the equation for $f_{n}$. This system gives the rules of all types of collisions. In the following we explain in detail how the rules of a P-N collision and a N-M collision are obtained from (2.5). The other collision rules can be derived similarly.

- P-N collisions. Collect all terms involving a P-N collision in (2.5),

$$
\begin{aligned}
& \left.\frac{\mathrm{d} f_{p}}{\mathrm{~d} t}\right|_{\mathrm{PN}}=Q^{-}\left(f_{n}\right) f_{p}, \\
& \left.\frac{\mathrm{d} f_{n}}{\mathrm{~d} t}\right|_{\mathrm{PN}}=Q^{+}\left(f_{p}, f_{n}\right)+Q^{+}\left(f_{n}, f_{p}\right)-Q^{-}\left(f_{p}\right) f_{n} .
\end{aligned}
$$

We can represent this collision by

$$
\mathbf{v}_{+}, \mathbf{w}_{-} \rightarrow 2 \mathbf{v}_{+}, \mathbf{v}_{-}^{\prime}, \mathbf{w}_{-}^{\prime},
$$

meaning that after the collision between a positive particle with velocity $\mathbf{v}_{+}$and a negative particle with velocity $\mathbf{w}_{-}$, the two particles $\mathbf{v}_{+}$and $\mathbf{w}_{-}$are removed, while four particles are created: two positive particles with (the same) velocity $\mathbf{v}_{+}$and two negative particles $\mathbf{v}_{-}^{\prime}$ and $\mathbf{w}_{-}^{\prime}$. Here prime denotes the post-collisional velocity in the usual definition (see, for example, $[7])$. 
- N-M collisions. Collect all terms involving a N-M collision,

$$
\begin{aligned}
\left.\frac{\mathrm{d} m}{\mathrm{~d} t}\right|_{\mathrm{NM}} & =0, \\
\left.\frac{\mathrm{d} f_{p}}{\mathrm{~d} t}\right|_{\mathrm{NM}} & =Q^{-}\left(f_{n}\right) m, \\
\left.\frac{\mathrm{d} f_{n}}{\mathrm{~d} t}\right|_{\mathrm{NM}} & =Q^{+}\left(m, f_{n}\right)+Q^{+}\left(f_{n}, m\right)-Q^{-}(m) f_{n} .
\end{aligned}
$$

This collision is represented by

$$
m, \mathbf{v}_{-} \rightarrow m, \mathbf{w}_{+}, \mathbf{v}_{-}^{\prime}, \mathbf{w}_{-}^{\prime},
$$

meaning that after collision the negative particle $\mathbf{v}_{-}$is removed, while a positive particle $\mathbf{w}$ sampled from $m$ and two negative particles $\mathbf{v}_{-}^{\prime}$ and $\mathbf{w}_{-}^{\prime}$ are created.

We summarize all the collision rules,

$$
\begin{array}{ll}
\text { P-P: } & \mathbf{v}_{+}, \mathbf{w}_{+} \rightarrow \mathbf{v}_{+}^{\prime}, \mathbf{w}_{+}^{\prime}, \\
\text { P-N: } & \mathbf{v}_{+}, \mathbf{w}_{-} \rightarrow 2 \mathbf{v}_{+}, \mathbf{v}_{-}^{\prime}, \mathbf{w}_{-}^{\prime}, \\
\text { N-N: } & \mathbf{v}_{-}, \mathbf{w}_{-} \rightarrow 2 \mathbf{v}_{-}, 2 \mathbf{w}_{-}, \mathbf{v}_{+}^{\prime}, \mathbf{w}_{+}^{\prime}, \\
\text { P-M: } & m, \mathbf{v}_{+} \rightarrow m, \mathbf{w}_{-}, \mathbf{v}_{+}^{\prime}, \mathbf{w}_{+}^{\prime}, \\
\text { N-M: } & m, \mathbf{v}_{-} \rightarrow m, \mathbf{w}_{+}, \mathbf{v}_{-}^{\prime}, \mathbf{w}_{-}^{\prime} .
\end{array}
$$

Similar to the traditional DSMC methods, like Bird's method or Nanbu-Babovsky method (see [15]), the total number of all collisions is $\mathcal{O}(\Delta t)$ in one time step. The numbers of each type of collision are proportional to the densities of participating particles.

The major problem in this algorithm is that all collisions (other than P-P collisions) create more particles than they delete. Since a number of $\mathcal{O}(\Delta t)$ extra particles are generated in each step, the total number grows approximately as

$$
\left.\left(N_{p}+N_{n}\right)\right|_{t+\Delta t}=\left.(1+c \Delta t)\left(N_{p}+N_{n}\right)\right|_{t}
$$

with some constant $c>0$. This means the particle number grows in the physical time scale, $N_{p}(t)+N_{n}(t)=\left(N_{p}(0)+N_{n}(0)\right) \mathcal{O}\left(e^{c t}\right)$.

\subsection{Coulomb collisions with negative particles}

Now we generalize the negative particle method to Coulomb collisions in a straightforward way. For Coulomb interaction, the formulation in (2.3) is not valid since the integrals in $Q^{+}$and $Q^{-}$are unbounded. Therefore one cannot directly apply the system (2.5) to Coulomb collisions.

Coulomb collisions are described by the Boltzmann type equation $(2.2)$ in which $Q(f, f)$ is the Landau-Fokker-Planck collision operator,

$$
Q(f, g)=\frac{A}{4} \frac{\partial}{\partial v_{i}} \int_{\mathbb{R}^{3}} u^{-3}\left(u^{2} \delta_{i j}-u_{i} u_{j}\right)\left(\frac{\partial}{\partial v_{j}}-\frac{\partial}{\partial w_{j}}\right) f(\mathbf{w}) g(\mathbf{v}) \mathrm{d} \mathbf{w}
$$

where $u=|\mathbf{v}-\mathbf{w}|, A=2 \pi\left(\frac{e^{2}}{2 \pi \varepsilon_{0} m}\right)^{2} \log \Lambda$, with $e$ the charge of an individual particle, $m$ its mass, $\varepsilon_{0}$ the permittivity of free space, and $\log \Lambda$ the Coulomb logarithm. Bobylev and Nanbu [5] showed that its solution can be approximated to first order in $\Delta t$ by

$$
f(\mathbf{v}, t+\Delta t)=\frac{1}{\rho} \iint_{\mathbb{R}^{3} \times \mathbb{S}^{2}} D\left(\frac{\mathbf{u} \cdot \mathbf{n}}{u}, A \frac{\Delta t}{u^{3}}\right) f\left(\mathbf{w}^{\prime}, t\right) f\left(\mathbf{v}^{\prime}, t\right) \mathrm{d} \mathbf{w} \mathrm{d} \mathbf{n} \doteq \frac{1}{\rho} P(f, f)
$$


with $\mathbf{u}=\mathbf{v}-\mathbf{w}, \rho$ the density of $f$. After normalization of (2.8) by dividing by $\rho$, the bilinear term $P(\hat{g}, \hat{f}) \geq 0$ gives the distribution of $\hat{f}$ due to collisions with $\hat{g}$. Here $\hat{f}$ is the normalized distribution of $f$.

Now we give two propositions on $P$. The proof is given in appendix for completeness.

Proposition 2.1. Two equalities hold for $P$,

1. For any normalized distributions $\hat{f}$ and $\hat{g}$,

$$
\int_{\mathbb{R}^{3}} P(\hat{g}, \hat{f}) \mathrm{d} \mathbf{v}=1
$$

2. For Maxwellian distribution $m$,

$$
P(\hat{m}, \hat{m})=\hat{m}(\mathbf{v}) .
$$

Here and after, the explicit dependence on $t$ is omitted in the notations for simplicity. The formula (2.8) can be decomposed in a similar way as in (2.5),

$$
\begin{aligned}
& m(\mathbf{v}, t+\Delta t)=\frac{\rho_{m}}{\rho} m(\mathbf{v}, t), \\
& f_{p}(\mathbf{v}, t+\Delta t)=\frac{\rho_{p}^{2}}{\rho} P\left(\hat{f}_{p}, \hat{f}_{p}\right)+\frac{\rho_{n}^{2}}{\rho} P\left(\hat{f}_{n}, \hat{f}_{n}\right)+\frac{\rho_{m} \rho_{p}}{\rho} P\left(\hat{m}, \hat{f}_{p}\right)+\frac{\rho_{p} \rho_{m}}{\rho} P\left(\hat{f}_{p}, \hat{m}\right), \\
& f_{n}(\mathbf{v}, t+\Delta t)=\frac{\rho_{p} \rho_{n}}{\rho} P\left(\hat{f}_{p}, \hat{f}_{n}\right)+\frac{\rho_{n} \rho_{p}}{\rho} P\left(\hat{f}_{n}, \hat{f}_{p}\right)+\frac{\rho_{m} \rho_{n}}{\rho} P\left(\hat{m}, \hat{f}_{n}\right)+\frac{\rho_{n} \rho_{m}}{\rho} P\left(\hat{f}_{n}, \hat{m}\right) .
\end{aligned}
$$

Noting that $\frac{\rho}{N_{\text {eff }}}=N_{m}+N_{p}-N_{n}$, the coefficients in front of each $P$ term in (2.11) suggest that after Coulomb collisions in one time step, the collection of positive particles consists of four parts:

- $\frac{N_{p}^{2}}{N_{m}+N_{p}-N_{n}}$ particles from P-P collisions;

- $\frac{N_{n}^{2}}{N_{m}+N_{p}-N_{n}}$ particles from N-N collisions;

- $\frac{N_{m} N_{p}}{N_{m}+N_{p}-N_{n}}$ particles from the positives particle involved in M-P collisions;

- $\frac{N_{m} N_{p}}{N_{m}+N_{p}-N_{n}}$ particles from the Maxwellian particles involved in M-P collisions.

Similarly the collection of negative particles also consists of four parts:

- $\frac{N_{p} N_{n}}{N_{m}+N_{p}-N_{n}}$ particles from the positive particles involved in P-N collisions;

- $\frac{N_{p} N_{n}}{N_{m}+N_{p}-N_{n}}$ particles from the negative particles involved in P-N collisions;

- $\frac{N_{m} N_{n}}{N_{m}+N_{p}-N_{n}}$ particles from the negative particles involved in M-N collisions;

- $\frac{N_{m} N_{n}}{N_{m}+N_{p}-N_{n}}$ particles from the Maxwellian particles involved in M-N collisions.

Hence after one time step, the total number of particles is

$$
\begin{aligned}
\left.\left(N_{p}+N_{n}\right)\right|_{t+\Delta t} & =\frac{1}{N_{m}+N_{p}-N_{n}}\left(N_{p}^{2}+N_{n}^{2}+2 N_{p} N_{n}+2 N_{p} N_{m}+2 N_{n} N_{m}\right) \\
& =\left.\left(1+\frac{N_{m}+2 N_{n}}{N_{m}+N_{p}-N_{n}}\right)\left(N_{p}+N_{n}\right)\right|_{t}
\end{aligned}
$$

Note that the coefficient, which is larger than one, does not depend on $\Delta t$. In other words, for any $t>0$, the total number $\left.\left(N_{p}+N_{n}\right)\right|_{t}$ grows to infinity, as $\Delta t \rightarrow 0$. The particle number grows on a 
numerical time scale. This is much worse than the rarefied gas collisions, where the total number grows on the physical time scale.

The underlying reason for this rapid increase is that for Coulomb collisions, all particles are collided in every time step $\Delta t$, no matter how small $\Delta t$ is. This is the effect of long range collisions between charged particles, in contrast to the short range collisions for charge-neutral particles. In the latter case, only $\mathcal{O}(\Delta t)$ collisions are performed.

\section{A New Negative Particle Method}

In this section we propose a new negative particle method for general bilinear operators, including the Boltzmann operator (2.3) for rarefied gas and the LFP operator (2.7) for Coulomb gas. The particle number grows on the physical time scale, even for the LFP equation. The idea is illustrated in Section 3.1 for general operator and applied to the LFP equation in Section 4.1. We give an error analysis in Section 3.3 and discuss the conservation of moments in Section 3.4. In Section 3.5 we propose an efficient method to reduce the number of particles to further accelerate this method.

\subsection{Collision Combinations and F-Particles}

Consider the spatial homogeneous Boltzmann type equation (2.2) with either rarefied gas collision (2.3) or Coulomb collision (2.7). Note that in either case $Q(f, g)$ is not symmetric between $f$ and $g$. It describes the changes in $g$ due to collisions with $f$. In other words, $f$ gives the source particles (or background particles) and $g$ gives the target particles. Similarly in the remainder of this paper, when we say an A-B collision, the A particle acts like a source (or background) and the B particle is the target. We only update the velocity of the $\mathrm{B}$ particle, while leaving the velocity of the $\mathrm{A}$ particle unchanged.

With the decomposition (2.1), the equation (2.2) can be reformulated

$$
\partial_{t} f=Q(f, f)=Q\left(f, f_{d}\right)+Q\left(f_{d}, m\right)+Q(m, m)=Q\left(f, f_{d}\right)+Q\left(f_{d}, m\right),
$$

and decomposed as

$$
\left\{\begin{array}{l}
\partial_{t} m=0 \\
\partial_{t} f_{d}=Q\left(f, f_{d}\right)+Q\left(f_{d}, m\right),
\end{array}\right.
$$

or equivalently

$$
\left\{\begin{array}{l}
\partial_{t} m=0, \\
\partial_{t} f_{p}=Q\left(f, f_{p}\right)+\left(Q\left(f_{p}-f_{n}, m\right)\right)_{+}, \\
\partial_{t} f_{n}=Q\left(f, f_{n}\right)+\left(Q\left(f_{p}-f_{n}, m\right)\right)_{-},
\end{array}\right.
$$

where $h_{ \pm}=\frac{1}{2}(|h| \pm h)$ is the positive/negative part. Again $Q(m, m)=0$ is dropped, meaning that the collisions between M-particles are omitted. For simplicity we leave the Maxwellian part $m(\mathbf{v}, t)$ invariant in time. One can also evolve $m$ to minimize the particle components. Compared to (2.4) or (2.5), the new equations (3.1) or (3.2) are a recombination of the collision terms.

The time evolution of positive particles includes two parts: First, the P-particles collide with particles sampled from the whole distribution $f$. Second, the source term $\left(Q\left(f_{p}-f_{n}, m\right)\right)_{+}$generates extra positive particles. The time evolution of negative particles is similar. Note that the collisions in the first parts $Q\left(f, f_{p}\right)$ and $Q\left(f, f_{n}\right)$ do not change the particle numbers, while the source terms create $\mathcal{O}\left(\Delta t\left(N_{p}+N_{n}\right)\right)$ particles in each time step. Therefore after one step the number of $\mathrm{P}$ and $\mathrm{N}$ particles grows by $(1+C \Delta t)$, with $C$ some constant. This result also holds for the Coulomb collision, as shown in Section 4.

Now the key problem is how to sample from $f$ directly for use in $Q\left(f, f_{p}\right)$ and $Q\left(f, f_{n}\right)$. A straightforward method is to recover $f_{p}$ and $f_{n}$ from the $\mathrm{P}$ and $\mathrm{N}$ particles by, for example, approximating the particles by Gaussians with small support, or counting particle numbers in a mesh in 
the phase space. All these methods are computationally expensive and inaccurate, especially for three dimensional velocity space.

To obtain samples from $f$ efficiently, we introduce another group of particles, called F-particles. F-particles are sampled from $f(t=0)$ initially and evolve according to the original equation (Boltzmann or LFP ). It is an independent collection of particles approximating the whole distribution $f$. The F-particles themselves give a numerical solution to the Boltzmann or LFP equations. When one needs to sample a particle from $f$ to collide with $\mathrm{P}$ and $\mathrm{N}$ particles in solving (3.2), one only needs to randomly pick up one sample from the pool of F-particles. The key ingredient is that F-particles are not necessary to be a very accurate approximation of $f$, so that $N_{\tilde{f}}$, the number of F-particles, could be small. In fact one only needs

$$
N_{\tilde{f}} \geq N_{p}+N_{n}
$$

which ensures there are enough F-particles to be used in the $Q\left(f, f_{p}\right)$ and $Q\left(f, f_{n}\right)$ collisions. We give a brief error analysis in Section 3.3 to show that the use of F-particles under the condition (3.3) introduces a negligible error.

F-particle gives a coarse approximation of $f$, while $\mathrm{P}$ and $\mathrm{N}$ particles are a finer approximation of $f-m$. From the view point of numerical methods for differential equations, F-particles act as a prediction of $f$, and $\mathrm{P}$ and $\mathrm{N}$ particles are a correction to $f-m$.

To summarize, we are solving the system

$$
\left\{\begin{aligned}
\partial_{t} \tilde{f} & =Q(\tilde{f}, \tilde{f}), \\
\partial_{t} m & =0, \\
\partial_{t} f_{d} & =Q\left(\tilde{f}, f_{d}\right)+Q\left(f_{d}, m\right),
\end{aligned}\right.
$$

or equivalently

$$
\left\{\begin{aligned}
\partial_{t} \tilde{f} & =Q(\tilde{f}, \tilde{f}), \\
\partial_{t} m & =0 \\
\partial_{t} f_{p} & =Q\left(\tilde{f}, f_{p}\right)+\left(Q\left(f_{p}-f_{n}, m\right)\right)_{+}, \\
\partial_{t} f_{n} & =Q\left(\tilde{f}, f_{n}\right)+\left(Q\left(f_{p}-f_{n}, m\right)\right)_{-} .
\end{aligned}\right.
$$

Here we use the notation $\tilde{f}$ to distinguish it from the finer solution $f=m+f_{p}-f_{n}$, which is the desired result.

Finally we would like to point out that in the evaluation of the source term $Q\left(f_{d}, m\right)=Q\left(f_{p}-\right.$ $\left.f_{n}, m\right)$, instead of looping over all P-particles and N-particles to represent $f_{p}$ and $f_{n}$, one only needs to use a subset of P-particles and N-particles with size $\mathcal{O}\left(\Delta t\left(N_{p}+N_{n}\right)\right)$. A brief error analysis is also given in Section 3.3.

Remark 3.1. A related work for the variable hard sphere (VHS) collisions has been done in [12]. In that work the quadratic term $Q\left(f_{p}-f_{n}, f_{p}-f_{n}\right)$ was neglected, and the remaining linearized VHS operator was decomposed into

$$
\begin{aligned}
\partial_{t} f_{p} & =\left[Q\left(f_{p}-f_{n}, m\right)+Q^{+}\left(m, f_{p}-f_{n}\right)\right]_{+}-Q^{-}(m) f_{p} \\
\partial_{t} f_{n} & =\left[Q\left(f_{p}-f_{n}, m\right)+Q^{+}\left(m, f_{p}-f_{n}\right)\right]_{-}-Q^{-}(m) f_{n} .
\end{aligned}
$$

Compared to this decomposition, there are two improvements in our formulation (3.5). First, (3.6) depends on the decomposition (2.3), which is not valid for the long range Coulomb collisions. While our formulation is applicable for general collisions. Second, (3.6) is based on the linearized operator, which is not accurate when the distribution is far away from the equilibrium, while in our formulation, the use of $\mathrm{F}$ particles makes it accurate in all regimes. 
We end this section with some discussions on the relation to the method described in Section 2.2 and to the TD method in $[6,17]$. dropped

Remark 3.2 (Relation to the method in Section 2.2). In the new system (3.5), many collisions terms in (2.5) or (2.11) are combined to reduce the number of additional particles For example, the extra particles generated from N-P collisions (i.e., $Q\left(f_{n}, f_{p}\right)$ in $\left.(2.5)\right)$ are eliminated by combining the N-P, P-P and M-P collisions (i.e., $Q\left(\tilde{f}, f_{p}\right)$ in $\left.(3.5)\right)$. In other words, instead of sampling N/P/M particles from $f_{n} / f_{p} / m$ and perform N-P/P-P/M-P collisions separately, one can sample particles from $f$ directly and perform F-P collisions. In addition, by sampling from $Q\left(f_{p}-f_{n}, m\right)$ directly, we also combine the $P-M$ and $N-M$ collisions.

Remark 3.3 (Relation to the TD method). Note that the term $Q\left(f_{p}-f_{n}, m\right)$ represents the change in the Maxwellian part after colliding with the $\mathrm{P}$ and $\mathrm{N}$ particles. Sampling positive and negative particles from $Q\left(f_{p}-f_{n}, m\right)$ is just the dethermalization process in the thermalization/dethermalization (TD) method. For the thermalization method:

- The dominant error source is the failed dethermalizations (see [17]), which does not vanish as $\Delta t \rightarrow 0$ or $N \rightarrow \infty$;

- Dethermalizing all Maxwellian particles involved in collisions with kinetic particles increases dramatically the number of kinetic particles. In fact the number grows unbounded as $\Delta t \rightarrow 0$, in a way that is similar to $(2.12)$.

In contrast, for our new formulation:

- We dethermalize all Maxwellian particles involved in collisions with kinetic particles. There is no error due to failed dethermalizations;

- By introducing negative particles and sampling from $Q\left(f_{p}-f_{n}, m\right)$ directly, we actually generate particles according to the net value of the dethermalized particle distribution. The numbers of $\mathrm{P}$ and $\mathrm{N}$ particles only increase by a factor of $\mathcal{O}(1+\Delta t)$ in one time step.

\subsection{Sample from the source term}

The next issue is the sampling of $\mathrm{P}$ and $\mathrm{N}$ particles from the source term $\Delta t Q\left(f_{p}-f_{n}, m\right)$ in $(3.5)$. We start with a general problem.

\subsubsection{Sample from a finite series}

Suppose we want to sample positive and negative particles from

$$
h(v)=\sum_{1 \leq k \leq n} h_{k}(v), \quad v \in \Omega
$$

with each term $h_{k}(v) \in L^{1}(\Omega)$. Note that $h_{k}(v)$ may be positive for some $v$ and negative for some other $v$. We further assume that for each $h_{k}$ we can find an upperbound

$$
\left|h_{k}(v)\right| \leq \bar{h}_{k}(v) \in L^{1}(\Omega),
$$

and that one can easily sample from $\bar{h}_{k}(v)$. Let $N_{\text {eff }}$ be the effective number, $\bar{h}(v)=\sum_{1 \leq k \leq n} \bar{h}_{k}(v)$, and

$$
N^{v}=\frac{1}{N_{\text {eff }}} \int \bar{h}(v) \mathrm{d} v .
$$

There are two methods to sample from $h(\mathbf{v})$. 
Method 1: First sample $N^{v}$ virtual particles from $\bar{h}(v)$. Then for each virtual particle with coordinate $v$, accept it with probability $\bar{h}(v)^{-1}|h(v)|$, and label it a positive or negative particle according to the sign of $h(v)$.

Method 2: For $i=1, \ldots, N^{v}$, choose one of the $\bar{h}_{k}$ with probability $\left(\int \bar{h}(v) \mathrm{d} v\right)^{-1}\left(\int \bar{h}_{k}(v) \mathrm{d} v\right)$. Then sample a virtual particle from $\bar{h}_{k}$. Denote its coordinate to be $v$. Accept this particle with probability $\bar{h}_{k}(v)^{-1}\left|h_{k}(v)\right|$, and label it a positive or negative particle according to the sign of $h(v)$.

We give a comparison of these two methods,

- Since the computation of $h(v)$ depends on evaluation of $\left\{h_{k}\right\}, k=1, \ldots, n$, Method 1 requires $\mathcal{O}\left(N^{v} n\right)$ evaluations, while Method 2 only requires $\mathcal{O}\left(N^{v}\right)$ evaluations. Thus for $n \gg 1$, Method 1 could be much slower than Method 2. However, as shown in Section 3.3, when $N^{v} \ll n$, one can approximate $h(v)$ with negligible error by evaluating a small random subset of $\left\{h_{k}\right\}$ terms.

- On the other hand, Method 1 creates less particles than Method 2, due to the fact that there may be cancellations in the sum of the $h_{k}$ 's. For example, if $h=h_{1}+h_{2}$ with $h_{2}(v)=-h_{1}(v)$ for all $v$, Method 1 generates no particles. However this difference is not significant if the supports of the different $h_{k}$ have very small overlap.

Furthermore, Method 2 can be improved by adding an extra rejection step: for each accepted particle $v$, reject it with probability

$$
p= \begin{cases}\min \left(1, \frac{\sum_{k}\left(h_{k}(v)\right)_{-}}{\sum_{k}\left(h_{k}(v)\right)_{+}}\right), & \text {if } v \text { is a positive particle } \\ \min \left(1, \frac{\sum_{k}\left(h_{k}(v)\right)_{+}}{\sum_{k}\left(h_{k}(v)\right)_{-}}\right), & \text {if } v \text { is a negative particle. }\end{cases}
$$

\subsubsection{Sample from the source term}

Denote

$$
\Delta m(\mathbf{v})=\Delta t Q\left(f_{p}-f_{n}, m\right),
$$

which describes the change in the Maxwellian part. Noting that $Q(\cdot, \cdot)$ is a linear operator in the first argument, and $f_{p}$ and $f_{n}$ are represented by particles, we have

$$
\begin{aligned}
\Delta m(\mathbf{v}) & \approx \Delta t Q\left(N_{\mathrm{eff}} \sum_{\mathbf{v}_{p}} \delta\left(\mathbf{v}-\mathbf{v}_{p}\right)-N_{\mathrm{eff}} \sum_{\mathbf{v}_{n}} \delta\left(\mathbf{v}-\mathbf{v}_{n}\right), m\right) \\
& =\sum_{\mathbf{v}_{p}} \delta m\left(\mathbf{v} ; \mathbf{v}_{p}\right)-\sum_{\mathbf{v}_{n}} \delta m\left(\mathbf{v} ; \mathbf{v}_{n}\right)
\end{aligned}
$$

where the summations are over all $\mathrm{P}$ and $\mathrm{N}$ particles, and

$$
\delta m\left(\mathbf{v} ; \mathbf{v}_{1}\right)=\Delta t N_{\mathrm{eff}} Q\left(\delta\left(\mathbf{v}-\mathbf{v}_{1}\right), m\right)
$$

describes the change in the Maxwellian part due to collisions with a particle of velocity $\mathbf{v}_{1}$.

Now one can apply one of the two methods described in Section 3.2.1 to sample from $\Delta m(\mathbf{v})$. The two key questions are

- How to evaluate $\delta m\left(\mathbf{v} ; \mathbf{v}_{1}\right)$, for any given velocity $\mathbf{v}$ and source particle with velocity $\mathbf{v}_{1}$.

- How to find an upper bound $\overline{\delta m}\left(\mathbf{v} ; \mathbf{v}_{1}\right)$ that is close to $\left|\delta m\left(\mathbf{v} ; \mathbf{v}_{1}\right)\right|$ and easy to sample.

We would like to point out that for general collision kernels the distribution $\delta m\left(\mathbf{v} ; \mathbf{v}_{1}\right)$ exhibits singularity near the source particle; i.e., at $\mathbf{v}=\mathbf{v}_{1}$. Therefore choosing $\overline{\delta m}(\mathbf{v})$ to be a constant distribution is inefficient. For different kernels the distribution $\delta m(\mathbf{v})$ could behave quite differently, making the choice of $\overline{\delta m}(\mathbf{v})$ highly problem dependent. In Section 4.2 we describe in detail the algorithm for the Coulomb collision. 


\subsection{Error Analysis}

In system (3.5) $\tilde{f}$ is the distribution represented by $N_{\tilde{f}}$ F-particles, where $N_{\tilde{f}}$ is a relatively small number satisfying the condition (3.3). In this section we show that approximating $Q\left(f, f_{p}\right)$ and $Q\left(f, f_{n}\right)$ by $Q\left(\tilde{f}, f_{p}\right)$ and $Q\left(\tilde{f}, f_{n}\right)$ does not deteriorate the accuracy of the whole algorithm.

Take the F-P collisions for example. First, since the $N_{\tilde{f}}$ F-particles evolve independently according to the original Boltzmann or LFP equation, $\tilde{f}(t)$ approximates the exact solution $f(t)$ with a statistical error of order $\left(N_{\tilde{f}}\right)^{-1 / 2}$, i.e.

$$
\tilde{f}(t)=f(t)+\mathcal{O}\left(\rho\left(N_{\tilde{f}}\right)^{-1 / 2}\right)
$$

where $\rho$ is the density of $f(t)$ and $\tilde{f}(t)$. For simplicity and in this section only, we assume $f$ is normalized, i.e. $\rho=1$. (Otherwise we can rescale the time variable $\tau=\rho t$, and rewrite the original equation (2.2) as $\frac{\partial}{\partial \tau} \hat{f}=Q(\hat{f}, \hat{f})$, with $\hat{f}$ the normalized distribution.)

The third equation in (3.5) gives

$$
\partial_{t} f_{p}=Q\left(\tilde{f}, f_{p}\right)+\left(Q\left(f_{p}-f_{n}, m\right)\right)_{+}=Q\left(f, f_{p}\right)+\left(Q\left(f_{p}-f_{n}, m\right)\right)_{+}+Q\left(\tilde{f}-f, f_{p}\right),
$$

which is the original equation in (3.2) with a drift term $Q\left(\tilde{f}-f, f_{p}\right)$. Note that solving the system (3.2) with $N_{p}$ P-particles introduces a statistical error $\mathcal{O}\left(\rho_{p}\left(N_{p}\right)^{-1 / 2}\right)$, while the magnitude of the drift term in (3.7) is $\mathcal{O}\left(\rho_{p}\left(N_{\tilde{f}}\right)^{-1 / 2}\right)$. As long as $N_{\tilde{f}} \geq N_{p}$, the drift error in approximating $f(t)$ by $\tilde{f}(t)$ is dominated by the sampling error of solving the whole system.

We also claim that evaluation of $\Delta m(\mathbf{v})$ in Method 1, in Section 3.2.1, does not require looping over all P-particles and N-particles. One only needs to randomly choose a subset of $\mathrm{P}$ and $\mathrm{N}$ particles of size $\mathcal{O}\left(\Delta t \rho_{m}\left(N_{p}+N_{n}\right)\right)$. Denote the distributions represented by this subset of particles by $\tilde{f}_{p}$ and $\tilde{f}_{n}$. Then

$$
\left(\tilde{f}_{p}-\tilde{f}_{n}\right)-\left(f_{p}-f_{n}\right)=\mathcal{O}\left(\left(\rho_{p}+\rho_{n}\right)\left(\Delta t \rho_{m}\left(N_{p}+N_{n}\right)\right)^{-1 / 2}\right)
$$

and

$$
\Delta t Q\left(\tilde{f}_{p}-\tilde{f}_{n}, m\right)=\Delta t Q\left(f_{p}-f_{n}, m\right)+\mathcal{O}\left(\Delta t \rho_{m}\left(\rho_{p}+\rho_{n}\right)\left(\Delta t \rho_{m}\left(N_{p}+N_{n}\right)\right)^{-1 / 2}\right) .
$$

As only $\mathcal{O}\left(\Delta t \rho_{m}\left(N_{p}+N_{n}\right)\right)$ particles are sampled from the first term on the right in (3.8), it introduces a statistical error of the same order as error term in (3.8).

A similar result was reported in [12], where only a small subset of particles were used in evaluating and sampling from a term of form $\int K\left(\mathbf{v}_{1}, \mathbf{v}\right)\left(f_{p}(\mathbf{v})-f_{n}(\mathbf{v})\right) \mathrm{d} \mathbf{v}$.

\subsection{Conservation of Moments}

Since we are not updating particles by pairs, the total momentum and energy are not conserved exactly in each realization. In addition, sampling from $\Delta m$ makes the total density not conserved either. Here we propose a simple way to enforce conservation.

After each time step, the conservation of total momentum and energy is enforced by shifting and rescaling the velocities of the P-particles and N-particles; the conservation of total density is enforced by adjusting $\rho_{m}$, the density of the Maxwellian part. The reason is that if one keeps $\rho_{m}$ invariant, one needs to remove some P-particles or N-particles for the sake of total density conservation. Since there are only $\mathcal{O}(\Delta t)$ extra particles created in one time step, removing some of them could lead to significant changes. 


\subsection{Control of Particle Number}

\subsubsection{Growth of particle number}

Since the total number of $\mathrm{P}$ and $\mathrm{N}$ particles increases by a factor of $(1+\mathcal{O}(\Delta t))$ in one time step, it grows exponentially in the worst case

$$
\left.\left(N_{p}+N_{n}\right)\right|_{t}=\left.\left.(1+C \Delta t)^{k}\left(N_{p}+N_{n}\right)\right|_{0} \rightarrow e^{C t}\left(N_{p}+N_{n}\right)\right|_{0}, \quad \text { as } \Delta t \rightarrow 0,
$$

here the number of time step is $k=t / \Delta t$. We would like to point out several facts,

- This is a significant improvement over the negative particle method described in Section 2.2 for Coulomb collisions, where the total number grows unbounded as $\Delta t \rightarrow 0$. In other words, it reduces the growth of particle number from the numerical scale to physical scale.

- Theoretically the total number does not grows as fast as $e^{C t}$. Note that $\tilde{f}$ approaches equilibrium as time increases, and $f_{p}$ and $f_{n}$ also approach equilibrium since they are colliding with $\tilde{f}$. Therefore both $\Delta t Q\left(f_{p}-f_{n}, m\right)$ in (3.5) and its approximation $\Delta m$ in (4.2) for Coulomb collisions are getting small as time evolves. Less and less particles are created and the total particle number stops increasing eventually.

- We could further reduce the number of the newly created particles by evolving the Maxwellian part. In other words, we could update the Maxwellian component to $m(\mathbf{v}, t+\Delta t)$, which may differ from $m(\mathbf{v}, t)$. Then we need to sample the extra $\mathrm{P}$ and $\mathrm{N}$ particles from the positive/negative part of

$$
\Delta m_{\bmod }(\mathbf{v})=\Delta t Q\left(f_{p}-f_{n}, m\right)-(m(\mathbf{v}, t+\Delta t)-m(\mathbf{v}, t)),
$$

instead of $\Delta t Q\left(f_{p}-f_{n}, m\right)$. By choosing a suitable $m(\mathbf{v}, t+\Delta t)$, more particles can be absorbed into the Maxwellian part and less particles are created. In this work we keep $m$ invariant for simplicity. The optimal choice of $m(\mathbf{v}, t+\Delta t)$ is left in future work. Here we refer the reader to [12] for some choices of $m(\mathbf{v}, t+\Delta t)$ in rarefied gas collisions.

\subsubsection{Reduction of particle number}

Although the particle number only grows in the physical scale, a particle reduction technique is still needed in our method for two reasons:

- The formula (3.3) needs to be valid for all the time. However $N_{\tilde{f}}$ is invariant while $N_{p}$ and $N_{n}$ grow with time and need to be reduced.

- Even if $N_{p}$ and $N_{n}$ are bounded, the efficiency of our method can be further improved by reducing the particle number, since the distributions $f_{p}$ and $f_{n}$ approach equilibrium as time evolves and they have overlap in velocity space.

The particle reduction does not need to be performed at each time step. It is only needed whenever (3.3) is violated. Several reduction methods could be used.

The most straightforward idea is particle cancellation, i.e., cancellation of a pair of particles with opposite signs if they are close enough in phase space, as in [3]. To do that, one places a mesh with grid size $\delta$ in velocity space and removes a pair of particles with opposite signs if they are located in the same cell. This method introduces an extra error of $\mathcal{O}(\delta)$. Small error is achieved only if $\delta$ is small, which makes the cancellation very inefficient since a large amount of particles are left uncancelled, especially in three dimension. This cancellation method only preserves the total density. Higher moments could be conserved by performing cancellation on more than two particles simultaneously, similar to the idea of particle merging in [13]. 
A second method is particle thermalization/dethermalization, which has been studied in $[6,17]$ based on the splitting (1.1). The thermalization process can reduce the number of particles by moving them into Maxwellian part. The entropy based method [17] gives first order accuracy on the thermalization process, which seems promising if combined with our method. We leave this in a future work.

In this work we propose a particle resampling method. We formulate an explicit approximation of $f_{p}$ and $f_{n}$ and replace the old $\mathrm{P} / \mathrm{N}$-particles by the new particles directly sampled from this approximation. The approximation is formed by selection of a complete basis set $\left\{\phi_{\mathbf{k}}\right\}$ and the following expansion and approximation:

$$
\begin{aligned}
f_{p}(\mathbf{v})-f_{n}(\mathbf{v}) & =\sum_{\mathbf{k}}\left\langle f_{p}-f_{n}, \phi_{\mathbf{k}}\right\rangle \phi_{\mathbf{k}}(\mathbf{v}) \approx \sum_{|\mathbf{k}|<K}\left\langle f_{p}-f_{n}, \phi_{\mathbf{k}}\right\rangle \phi_{\mathbf{k}}(\mathbf{v}) \\
& \approx N_{\mathrm{eff}} \sum_{|\mathbf{k}|<K}\left(\sum_{\mathbf{v}_{p}} \phi_{\mathbf{k}}\left(\mathbf{v}_{p}\right)-\sum_{\mathbf{v}_{n}} \phi_{\mathbf{k}}\left(\mathbf{v}_{n}\right)\right) \phi_{\mathbf{k}}(\mathbf{v}) .
\end{aligned}
$$

Here $\langle\cdot, \cdot\rangle$ is the inner product, and $K$ is a cutoff parameter. To sample from $\left(f_{p}-f_{n}\right)$, one can apply Method 1 described in Section 3.2.1 to the finite series (3.11).

A simple choice of $\left\{\phi_{\mathbf{k}}\right\}$ is the Fourier basis $\phi_{\mathbf{k}}(\mathbf{v})=\exp (-i \mathbf{k} \cdot \mathbf{v})$. After calculating coefficients $\left\langle f_{p}-f_{n}, \phi_{\mathbf{k}}\right\rangle$, one can perform the fast inverse Fourier transform and obtain a function, denoted by $\left(\tilde{f}_{p}-\tilde{f}_{n}\right)$, on a mesh with $K^{3}$ nodes. Then one can define the upper bound $F(\mathbf{v}) \gtrsim\left|\tilde{f}_{p}-\tilde{f}_{n}\right|$ which serves the role of $\bar{h}$ in Method 1 from Section 3.2.1.

The total cost is $\mathcal{O}\left(K^{3}\left(N_{p}+N_{n}\right)\right)$ for evaluation of the expansion coefficients in (3.11). Note that this particle resampling is computationally expensive compared to the other parts of simulation, i.e., the F-F, P-F, N-F collisions and the sampling from $\Delta t Q\left(f_{p}-f_{n}, m\right)$. The particle resampling is only performed a few times in the whole simulation; e.g., whenever (3.3) is violated.

Next, after new $\mathrm{P}$ and $\mathrm{N}$ particles are resampled, it is not necessary to keep all the $\mathrm{F}$ particles, since one only needs (3.3) to be valid after the resampling, as demonstrated in Section 3.3. Therefore we can randomly pick a subset of the original $\mathrm{F}$ particles and continue the evolution.

To summarize, the resampling algorithm is

- Initialization: Choose a constant $\alpha \gtrsim 1$. Sample $N_{\tilde{f}}=\alpha\left(N_{p}+N_{n}\right)$ F-particles from the initial distribution $f(t=0)$.

- In each time step:

- Solve (3.5) for one step.

- If $\left(N_{p}+N_{n}\right) \geq N_{\tilde{f}}$, apply the particle resampling method. Denote $N_{p}$ and $N_{n}$ to be the numbers of the newly resampled $\mathrm{P}$ and $\mathrm{N}$ particles. Update $N_{\tilde{f}}=\alpha\left(N_{p}+N_{n}\right)$ and select new F-particles by randomly choosing $N_{\tilde{f}}$ particles from the original F-particles. Note that in the spatially homogeneous step, $N_{\tilde{f}}$ is decreasing in time.

Remark 3.4 (On the error). The error in resampling consists of two parts: (a) discretization error due to the cut off at $K$-th term; (b) statistical error in the expansion coefficients from the use of $N_{p}$ and $N_{n}$ particles. The cut off error is very small since the distributions $f_{p}$ and $f_{n}$ are smooth in the velocity space due to Coulomb collisions (see [19]). The statistical error is $\mathcal{O}\left(\left(\rho_{p}+\rho_{n}\right)\left(N_{p}+N_{n}\right)^{-1 / 2}\right)$, which is not larger than the statistical error in F-P and F-N collisions.

With the existence of an external force or boundary condition, the distributions $f_{p}$ and $f_{n}$ might become non-smooth as time evolves. Then interpolation with a global basis could lead to a relatively large error, and one could use some local basis to improve the efficiency. As the resampling process is completely independent from the rest of the simulation method, one can easily replace it with a different method adapted to the solution behavior. 
Remark 3.5. The constant $\alpha$ is crucial to efficiency of the method. On one hand, a large $\alpha$ slows down the step of solving (3.5) and the following particle resampling, since there can be more $\mathrm{P}$ and $\mathrm{N}$ particles before being resampled. On the other hand, a large $\alpha$ can accelerate this step since it leads to low frequency of performing particle resampling, which is computationally expensive. Moreover, a time-dependent $\alpha$ might also increase the efficiency. The choice of an optimal $\alpha$ will be studied in the future work.

\section{Application to Coulomb collisions}

Now we apply the ideas from the previous last to the TA method or Nanbu's method for Coulomb collisions.

\subsection{Reformulation of Coulomb collisions}

Stating from the framework of Bobylev-Nanbu's formulation (2.8), we recombine (2.8) as in (3.1) to get

$$
\begin{aligned}
f(\mathbf{v}, t+\Delta t) & =\frac{1}{\rho} P(f, f) \\
& =\frac{1}{\rho} P(m, m)+\frac{1}{\rho} P\left(f, f_{p}\right)-\frac{1}{\rho} P\left(f, f_{n}\right)+\frac{1}{\rho} P\left(f_{p}-f_{n}, m\right) \\
& =m(\mathbf{v})+\frac{1}{\rho} P\left(f, f_{p}\right)-\frac{1}{\rho} P\left(f, f_{n}\right)+\left(\frac{1}{\rho} P\left(f_{p}-f_{n}, m\right)-\frac{\rho_{p}-\rho_{n}}{\rho} m(\mathbf{v})\right),
\end{aligned}
$$

where we have used the fact $\rho=\rho_{m}+\rho_{p}-\rho_{n}$, and $P(m, m)=\rho_{m} m$ due to (2.10).

Next expand this equation as in (3.5) to get

$$
\begin{aligned}
\tilde{f}(\mathbf{v}, t+\Delta t) & =\frac{1}{\rho} \iint_{\mathbb{R}^{3} \times \mathbb{S}^{2}} D \tilde{f}\left(\mathbf{w}^{\prime}\right) \tilde{f}\left(\mathbf{v}^{\prime}\right) \mathrm{d} \mathbf{w} \mathrm{d} \mathbf{n}, \\
m(\mathbf{v}, t+\Delta t) & =m(\mathbf{v}, t), \\
f_{p}(\mathbf{v}, t+\Delta t) & =\frac{1}{\rho} \iint_{\mathbb{R}^{3} \times \mathbb{S}^{2}} D \tilde{f}\left(\mathbf{w}^{\prime}\right) f_{p}\left(\mathbf{v}^{\prime}\right) \mathrm{d} \mathbf{w} \mathrm{d} \mathbf{n}+(\Delta m(\mathbf{v}))_{+}, \\
f_{n}(\mathbf{v}, t+\Delta t) & =\frac{1}{\rho} \iint_{\mathbb{R}^{3} \times \mathbb{S}^{2}} D \tilde{f}\left(\mathbf{w}^{\prime}\right) f_{n}\left(\mathbf{v}^{\prime}\right) \mathrm{d} \mathbf{w} \mathrm{d} \mathbf{n}+(\Delta m(\mathbf{v}))_{-},
\end{aligned}
$$

where

$$
\Delta m(\mathbf{v})=\frac{1}{\rho} \iint_{\mathbb{R}^{3} \times \mathbb{S}^{2}} D\left(f_{p}\left(\mathbf{w}^{\prime}\right)-f_{n}\left(\mathbf{w}^{\prime}\right)\right) m\left(\mathbf{v}^{\prime}\right) \mathrm{d} \mathbf{w} \mathrm{d} \mathbf{n}-\frac{\rho_{p}-\rho_{n}}{\rho} m(\mathbf{v})
$$

is the change in Maxwellian part $m$ due to the collisions with particles sampled from $f_{p}$ and $f_{n}$. $\Delta m$ is an approximation of $\Delta t Q\left(f_{p}-f_{n}, m\right)$ in (3.5) (or equivalently, of $\rho^{-1} P\left(f_{p}-f_{n}, m\right)$ in (2.8)),

$$
\Delta m=\Delta t Q\left(f_{p}-f_{n}, m\right)+\mathcal{O}\left(\Delta t^{2}\right) .
$$

Therefore (4.1) gives a first order approximation to (3.5) (or (2.8)).

According to (4.1), the F-particles are updated independently by performing F-F collisions. The Maxwellian part $m$ is not changed. At $t+\Delta t$, the P-particles come from two parts: (1) $N_{p}$ particles come from collisions of old P-particles with F-particles; and (2) an additional set of $\mathcal{O}\left(N_{p} \Delta t\right)$ particles are sampled from the positive part of $\Delta m(\mathbf{v})$. The new N-particles are obtained similarly. As expected, after one step the numbers of $\mathrm{P}$ and $\mathrm{N}$ particles grow by $(1+C \Delta t)$, with $C$ a constant. This is a significant improvement compared to (2.12), where the growth factor is a constant independent of $\Delta t$ and larger than one. 


\subsection{Sample from the source term}

In this section we describe how to sample $\mathrm{P}$ and $\mathrm{N}$ particles from the source term $\Delta t Q\left(f_{p}-f_{n}, m\right)$ in (3.5). For the Coulomb collisions, it is approximated by $\Delta m(\mathbf{v})$ in (4.2), given by

$$
\begin{aligned}
\Delta m(\mathbf{v}) & \approx \frac{N_{\mathrm{eff}}}{\rho} \iint_{\mathbb{R}^{3} \times \mathbb{S}^{2}} D m\left(\mathbf{v}^{\prime}\right)\left(\sum_{\mathbf{v}_{p}} \delta\left(\mathbf{w}^{\prime}-\mathbf{v}_{p}\right)-\sum_{\mathbf{v}_{n}} \delta\left(\mathbf{w}^{\prime}-\mathbf{v}_{n}\right)\right) \mathrm{d} \mathbf{w} \mathrm{d} \mathbf{n}-\frac{\rho_{p}-\rho_{n}}{\rho} m(\mathbf{v}) \\
& =\frac{N_{\mathrm{eff}}}{\rho} \sum_{\mathbf{v}_{p}} \delta m\left(\mathbf{v} ; \mathbf{v}_{p}\right)-\frac{N_{\mathrm{eff}}}{\rho} \sum_{\mathbf{v}_{n}} \delta m\left(\mathbf{v} ; \mathbf{v}_{n}\right),
\end{aligned}
$$

where

$$
\delta m\left(\mathbf{v} ; \mathbf{v}_{1}\right)=\iint_{\mathbb{R}^{3} \times \mathbb{S}^{2}} D\left(\frac{(\mathbf{v}-\mathbf{w}) \cdot \mathbf{n}}{|\mathbf{v}-\mathbf{w}|}, A \frac{\Delta t}{|\mathbf{v}-\mathbf{w}|^{3}}\right) m\left(\mathbf{v}^{\prime}\right) \delta\left(\mathbf{w}^{\prime}-\mathbf{v}_{1}\right) \mathrm{d} \mathbf{w} \mathrm{d} \mathbf{n}-m(\mathbf{v})
$$

describes the change in $m$ due to collisions with particles with velocity $\mathbf{v}_{1}$.

As discussed in Section 3.2, the two main questions are (a) how to evaluate $\delta m\left(\mathbf{v} ; \mathbf{v}_{1}\right)$, for any $\mathbf{v}$ with given source particle $\mathbf{v}_{1}$ and (b) how to find a suitable upper bound $\overline{\delta m}\left(\mathbf{v} ; \mathbf{v}_{1}\right)$. These questions are answered in Section 4.2.1, with the detailed proof given in Appendix C. The sampling algorithm is summarized in Section 4.2.2 for reader's convenience.

\subsubsection{Main Results}

Note that $\delta m\left(\mathbf{v} ; \mathbf{v}_{1}\right)$ is a five-dimension integral, which is expensive to evaluate. In Appendix C, we reduce this to a two dimensional integral and further approximate it by a one dimensional integral. The results can be summarized as,

Proposition 4.1 (Evaluation and approximation of $\delta m\left(\mathbf{v} ; \mathbf{v}_{1}\right)$ ).

- $\delta m\left(\mathbf{v} ; \mathbf{v}_{1}\right)$ can be simplified as

$$
\delta m\left(\mathbf{v}_{0} ; \mathbf{v}_{1}\right)=\int_{-1}^{1}\left(\frac{1+\mu}{2}\right)^{-3 / 2} 2 \pi \tilde{D}(\mu, \tau(\mu))\left(\frac{1}{2 \pi} \int m(\mathbf{v}(\mu, \phi)) \mathrm{d} \phi\right) \mathrm{d} \mu-m\left(\mathbf{v}_{0}\right),
$$

with

$$
\begin{aligned}
& \mathbf{v}(\mu, \phi)=\mathbf{v}_{0}+\left|\mathbf{v}_{0}-\mathbf{v}_{1}\right| \sqrt{\frac{1-\mu}{1+\mu}}\left(\hat{\mathbf{s}}_{1} \cos \phi+\hat{\mathbf{s}}_{2} \sin \phi\right), \\
& \tau(\mu)=A \Delta t\left|\mathbf{v}-\mathbf{v}_{1}\right|^{-3}=A \frac{\Delta t}{\left|\mathbf{v}_{0}-\mathbf{v}_{1}\right|^{3}}((1+\mu) / 2)^{3 / 2}
\end{aligned}
$$

Here $\hat{\mathbf{s}}_{1}$ and $\hat{\mathbf{s}}_{2}$ are two orthogonal unit vectors in the plane perpendicular to $\left(\mathbf{v}_{0}-\mathbf{v}_{1}\right)$. The collision kernel $\tilde{D}(\mu, \tau)$ corresponds to different numerical methods for performing regular Coulomb collisions. Several choices of $\tilde{D}$ are summarized in Appendix B.

- $\delta m\left(\mathbf{v}_{0} ; \mathbf{v}_{1}\right)$ can be approximated by a one dimensional integral,

$$
\delta m\left(\mathbf{v}_{0} ; \mathbf{v}_{1}\right) \approx m\left(\mathbf{v}_{0}\right)\left[\int_{-1}^{1}\left(\frac{1+\mu}{2}\right)^{-3 / 2} 2 \pi \tilde{D}(\mu, \tau) e^{-\varepsilon^{2}}\left(1+\left(\varepsilon v_{\perp}\right)^{2}+\frac{1}{4}\left(\varepsilon v_{\perp}\right)^{4}\right) \mathrm{d} \mu-1\right]
$$

with $\tau=\tau(\mu)$ given in (4.7) and

$$
\varepsilon=\varepsilon(\mu)=\frac{\left|\mathbf{v}_{0}-\mathbf{v}_{1}\right|}{\sqrt{2 T_{m}}} \sqrt{\frac{1-\mu}{1+\mu}}
$$




$$
v_{\perp}=\frac{1}{\sqrt{2 T_{m}}}\left|\mathbf{v}_{0}-\mathbf{u}_{m}-\left(\left(\mathbf{v}_{0}-\mathbf{u}_{m}\right) \cdot \frac{\mathbf{v}_{0}-\mathbf{v}_{1}}{\left|\mathbf{v}_{0}-\mathbf{v}_{1}\right|}\right) \frac{\mathbf{v}_{0}-\mathbf{v}_{1}}{\left|\mathbf{v}_{0}-\mathbf{v}_{1}\right|}\right| .
$$

Here $\mathbf{u}_{m}$ and $T_{m}$ are the macroscopic velocity and temperature of the Maxwellian part $m$. This is a first order approximation in $\Delta t$; i.e., the error in approximation (4.8) is $\mathcal{O}\left(\Delta t^{2}\right)$.

- For TA method, (4.8) can be reformulated

$$
\delta m\left(\mathbf{v}_{0} ; \mathbf{v}_{1}\right) \approx m\left(\mathbf{v}_{0}\right)\left[\frac{2 r^{1 / 2}}{\sqrt{\pi}} \int_{0}^{\infty}\left(1+\zeta^{2}\right)^{9 / 4} e^{-r \zeta^{2}\left(1+\zeta^{2}\right)^{3 / 2}} e^{-s^{2} \zeta^{2}}\left(1+s^{2} \zeta^{2}+\frac{1}{4} s^{4} \zeta^{4}\right) \mathrm{d} \zeta-1\right]
$$

with $r=\frac{\left|\mathbf{v}_{0}-\mathbf{v}_{1}\right|^{3}}{2 A \Delta t}$ and $s=\frac{\left|\mathbf{v}_{0}-\mathbf{v}_{1}\right| v_{\perp}}{\sqrt{2 T_{m}}}$. This integral can be numerically computed over $\zeta \in$ $\left[0, \zeta_{\max }\right]$ with the cut off $\zeta_{\max }$ approximately given by

$$
\zeta_{\max }=\zeta_{\max }(r)= \begin{cases}e^{-0.0026(\log r)^{2}-0.4150 \log r+0.9154}, & \text { if } r \geq 1, \\ e^{-0.0006(\log r)^{2}-0.2242 \log r+0.4711}, & \text { otherwise. }\end{cases}
$$

Then in Appendix D we show how to find the upper bounds of $\delta m\left(\mathbf{v} ; \mathbf{v}_{1}\right)$ and $\Delta m(\mathbf{v})$. First we introduce the following notation:

Definition 4.2. Let $\delta m=\delta m_{+}-\delta m_{-}$, with $\delta m_{ \pm}$the positive/negative part of $\delta m$. Denote $\Delta m=\Delta m_{+}-\Delta m_{-}$in (4.3), with

$$
\begin{aligned}
& \Delta m_{+}(\mathbf{v})=\frac{N_{\mathrm{eff}}}{\rho} \sum_{\mathbf{v}_{p}} \delta m_{+}\left(\mathbf{v} ; \mathbf{v}_{p}\right)-\frac{N_{\mathrm{eff}}}{\rho} \sum_{\mathbf{v}_{n}} \delta m_{+}\left(\mathbf{v} ; \mathbf{v}_{n}\right) \\
& \Delta m_{-}(\mathbf{v})=\frac{N_{\mathrm{eff}}}{\rho} \sum_{\mathbf{v}_{p}} \delta m_{-}\left(\mathbf{v} ; \mathbf{v}_{p}\right)-\frac{N_{\mathrm{eff}}}{\rho} \sum_{\mathbf{v}_{n}} \delta m_{-}\left(\mathbf{v} ; \mathbf{v}_{n}\right) .
\end{aligned}
$$

Note that $\Delta m_{ \pm}(\mathbf{v})$ are not the positive/negative part of $\Delta m(\mathbf{v})$ and they are not always nonnegative. Detailed analysis suggests that $\delta m_{+}$and $\delta m_{-}$have quite different behaviors. It is easier to sample from $\Delta m_{+}$and $\left(-\Delta m_{-}\right)$separately instead of sampling from $\Delta m$.

We also define two positive constants $\alpha_{l}=\alpha_{l}\left(\Delta t, T_{m}\right)$ and $\alpha_{u}=\alpha_{u}\left(\Delta t, T_{m}, \beta\right)$ by

Definition $4.3\left(\alpha_{l}\right.$ and $\left.\alpha_{u}\right)$.

$$
\begin{gathered}
-\alpha_{l}=\min _{u_{01}} \int_{-1}^{1}\left(\frac{1+\mu}{2}\right)^{-3 / 2} 2 \pi \tilde{D}(\mu, \tau) e^{-\varepsilon^{2}} \mathrm{~d} \mu-1, \\
\alpha_{u}=c_{0}+\beta^{2} c_{1}+\beta^{4} c_{2},
\end{gathered}
$$

where

$$
\begin{aligned}
& c_{0}=\max _{u_{01}} u_{01}^{2}\left(\int_{-1}^{1}\left(\frac{1+\mu}{2}\right)^{-3 / 2} 2 \pi \tilde{D}(\mu, \tau) e^{-\varepsilon^{2}} \mathrm{~d} \mu-1\right), \\
& c_{1}=\max _{u_{01}} u_{01}^{2} \int_{-1}^{1}\left(\frac{1+\mu}{2}\right)^{-3 / 2} 2 \pi \tilde{D}(\mu, \tau) e^{-\varepsilon^{2}} \varepsilon^{2} \mathrm{~d} \mu, \\
& c_{2}=\max _{u_{01}} u_{01}^{2} \int_{-1}^{1}\left(\frac{1+\mu}{2}\right)^{-3 / 2} 2 \pi \tilde{D}(\mu, \tau) e^{-\varepsilon^{2}} \varepsilon^{4} \mathrm{~d} \mu,
\end{aligned}
$$

in which $u_{01}:=\left|\mathbf{v}_{0}-\mathbf{v}_{1}\right|, \tau=\tau(\mu)$ is given in (4.7) and $\varepsilon=\varepsilon(\mu)$ is given in (4.9). Here $\beta$ is a suitable "cut off" on the tail of a normal distribution; i.e., the mass $\int_{|\mathbf{v}| \geq \beta}(2 \pi)^{-3 / 2} \exp \left(-|\mathbf{v}|^{2} / 2\right) \mathrm{d} \mathbf{v}$ is negligible. In practice we take $\beta=3$. 
The main results on the upper bounds are

Proposition 4.4 (Upper bounds).

- For $\delta m_{ \pm}\left(\mathbf{v} ; \mathbf{v}_{1}\right)$,

$$
\begin{aligned}
& 0 \leq\left|\mathbf{v}-\mathbf{v}_{1}\right|^{2} \delta m_{+}\left(\mathbf{v} ; \mathbf{v}_{1}\right) \leq \alpha_{u} m(\mathbf{v}) \\
& 0 \leq \delta m_{-}\left(\mathbf{v} ; \mathbf{v}_{1}\right) \leq \alpha_{l} m(\mathbf{v})
\end{aligned}
$$

- For $\Delta m_{ \pm}(\mathbf{v})$,

$$
\begin{aligned}
& \left|\Delta m_{+}(\mathbf{v})\right| \leq \overline{\Delta m_{+}}(\mathbf{v}) \doteq \frac{\alpha_{u} N_{e f f}}{\rho} \frac{\rho_{m}}{\left(2 \pi T_{m}\right)^{3 / 2}}\left(\sum_{\mathbf{v}_{p}} \frac{1}{\left|\mathbf{v}-\mathbf{v}_{p}\right|^{2}}+\sum_{\mathbf{v}_{n}} \frac{1}{\left|\mathbf{v}-\mathbf{v}_{n}\right|^{2}}\right), \\
& \left|\Delta m_{-}(\mathbf{v})\right| \leq \overline{\Delta m_{-}}(\mathbf{v}) \doteq \frac{\alpha_{l} N_{\text {eff }}}{\rho}\left(N_{p}+N_{n}\right) m(\mathbf{v}) .
\end{aligned}
$$

\subsubsection{Algorithm of sampling from $\Delta m(\mathrm{v})$}

Here is an algorithm for sampling from $\Delta m(\mathbf{v})$, based on the results in Section 4.2.1.

Since we have derived the upper bounds of $\left|\Delta m_{ \pm}\right|$, we can sample particles from $\Delta m_{+}$and $\left(-\Delta m_{-}\right)$separately, instead of directly from $\Delta m$.

We apply the two methods described in Section 3.2.1 on $\Delta m_{+}$and $\left(-\Delta m_{-}\right)$. For $\left(-\Delta m_{-}\right)$, we apply Method 1, since each $\delta m_{-}$term has relatively large support and cancellation between different $\delta m_{-}$terms leads to a small number of particles generated in Method 1 . For $\Delta m_{+}$, the cancellation is not significant since the singularity leads to a small support of each $\delta m_{+}$term, so that find Method 2 is more efficient.

Note that $\alpha_{l}$ and $\alpha_{u}$, which only depend on $\Delta t$ and $T_{m}$, can be pre-computed from (4.13) and (4.14)-(4.15), and updated only when the Maxwellian temperature $T_{m}$ is changed. In (4.14) we use $\beta=3$.

Algorithm for sampling from $\left(-\Delta m_{-}\right)$

- Let

$$
N_{n}^{v}=\frac{1}{N_{\text {eff }}} \int_{\mathbb{R}^{3}} \overline{\Delta m_{-}}(\mathbf{v}) \mathrm{d} \mathbf{v}=\frac{\alpha_{l} \rho_{m}}{\rho}\left(N_{p}+N_{n}\right) .
$$

Sample $N_{n}^{v}$ virtual particles from $m(\mathbf{v})$.

- Calculate all values $\delta m\left(\mathbf{v}, \mathbf{v}_{p}\right)$ and $\delta m\left(\mathbf{v}, \mathbf{v}_{n}\right)$ that go into sums for $\Delta m_{+}$and $\Delta m_{-}$.

- For each virtual particle with velocity $\mathbf{v}$, evaluate $\Delta m_{-}(\mathbf{v})$ from (4.12) and (4.8) (or (4.11) for TA method). Accept this particle with rate $\left|\Delta m_{-}(\mathbf{v})\right| \overline{\Delta m_{-}}(\mathbf{v})$. If $\Delta m_{-}(\mathbf{v})>0$, label it a negative particle; otherwise label it positive.

Note that the sign of the accepted particle $\mathbf{v}$ is opposite to the sign of $\Delta m_{-}(\mathbf{v})$.

Sampling from $\Delta m_{+}(\mathbf{v})$ using Method 2 requires values of $\int \overline{\Delta m_{+}}(\mathbf{v}) \mathrm{d} \mathbf{v}$. Note that the integral of (4.17) involves integrals $\int\left|\mathbf{v}-\mathbf{v}_{1}\right|^{-2} \mathrm{~d} \mathbf{v}$ that unbounded in the whole $3 \mathrm{D}$ velocity space. In computation one can assume the support of $\delta m_{+}$is bounded; i.e., we only sample particles with velocity $\mathbf{v}$ satisfying

$$
\left|\mathbf{v}-\mathbf{v}_{1}\right|<r_{\max }
$$

for some constant $r_{\max }$. Then $\int_{\left|\mathbf{v}-\mathbf{v}_{1}\right|<r_{\max }}\left|\mathbf{v}-\mathbf{v}_{1}\right|^{-2} \mathrm{~d} \mathbf{v}=4 \pi r_{\max }$.

Algorithm for sampling from $\Delta m_{+}$ 
- Let

$$
N_{p}^{v}=\frac{1}{N_{\text {eff }}} \int \overline{\Delta m_{+}}(\mathbf{v}) \mathrm{d} \mathbf{v}=\frac{4 \pi r_{\max } \alpha_{u}}{\rho} \frac{\rho_{m}}{\left(2 \pi T_{m}\right)^{3 / 2}}\left(N_{p}+N_{n}\right)
$$

- For $k_{p}=1, \ldots, N_{p}^{v}$, randomly pick a number $q_{0} \in[0,1]$.

If $q_{0} \leq \frac{N_{p}}{N_{p}+N_{n}}$,

- Randomly pick a P particle $\mathbf{v}_{p}$.

- Choose $q_{1}, q_{2}, q_{3} \in[0,1]$ uniformly. Let $\mathbf{v}=\mathbf{v}_{p}+r(\sin \theta \cos \phi, \sin \theta \sin \phi, \cos \theta)$, where

$$
r=q_{1} r_{\max }, \quad \cos \theta=2 q_{2}-1, \quad \sin \theta=\sqrt{1-\cos ^{2} \theta}, \quad \phi=2 \pi q_{3} .
$$

- Pick a number $q_{4} \in[0,1]$ uniformly. If $q_{4} \leq \frac{m(\mathbf{v})}{\rho_{m}\left(2 \pi T_{m}\right)^{-3 / 2}}$, continue to next step. Otherwise reject this particle.

- Evaluate $\delta m_{+}\left(\mathbf{v} ; \mathbf{v}_{p}\right)$ from (4.8). Accept this particle with rate $\left|\delta m_{+}\left(\mathbf{v} ; \mathbf{v}_{p}\right)\right| /\left(\alpha_{u} \rho_{m} \mid \mathbf{v}-\right.$ $\left.\mathbf{v}_{p}\right|^{-2}$ ) and label it a positive particle. Otherwise reject this particle.

Otherwise (i.e., if $q_{0}>\frac{N_{p}}{N_{p}+N_{n}}$ ),

- Randomly pick a N particle $\mathbf{v}_{n}$.

- Choose $q_{1}, q_{2}, q_{3} \in[0,1]$ uniformly. Let $\mathbf{v}=\mathbf{v}_{n}+r(\sin \theta \cos \phi, \sin \theta \sin \phi, \cos \theta)$, with $r, \theta$ and $\phi$ defined in (4.21).

- Pick a number $q_{4} \in[0,1]$ uniformly. If $q_{4} \leq \frac{m(\mathbf{v})}{\rho_{m}\left(2 \pi T_{m}\right)^{-3 / 2}}$, continue to next step. Otherwise reject this particle.

- Evaluate $\delta m_{+}\left(\mathbf{v} ; \mathbf{v}_{n}\right)$ from (4.8). Accept this particle with rate $\left|\delta m_{+}\left(\mathbf{v} ; \mathbf{v}_{n}\right)\right| /\left(\alpha_{u} \rho_{m} \mid \mathbf{v}-\right.$ $\left.\mathbf{v}_{n}\right|^{-2}$ ) and label it a negative particle. Otherwise reject this particle.

We end this section with some remarks,

Remark 4.5. As illustrated in Section 3.3, we only need to loop over a subset of $\mathrm{P}$ and $\mathrm{N}$ particles in evaluating $\Delta m_{-}(\mathbf{v})$. One needs to change the effective number $N_{\text {eff }}$ accordingly:

$$
N_{\mathrm{eff}}^{\text {subset }}=\frac{N_{p} N_{\mathrm{eff}}}{N_{p}^{\text {subset }}}
$$

Remark 4.6 (On the choice of $r_{\max }$ ). The parameter $r_{\max }$ in the assumption (4.19) determines the number of virtual particles in (4.20). In this work we take $r_{\max }=3 \sqrt{2 T_{m}}$, which gives quite satisfactory results. Two observations from numerical simulations support this choice: (1) If the source particle $\mathbf{v}_{1}$ is close to $\mathbf{u}_{m}$, the center of Maxwellian part $m$, the support of $\delta m_{+}\left(\mathbf{v} ; \mathbf{v}_{1}\right)$ is very small (far smaller than $3 \sqrt{2 T_{m}}$ ). (2) If the source particle $\mathbf{v}_{1}$ is far away from $\mathbf{u}_{m}$, the function $\delta m_{+}\left(\mathbf{v} ; \mathbf{v}_{1}\right)$ itself is very small.

In fact we only need $r_{\max }$ big enough such that

$$
\operatorname{supp}\left(\delta m_{+}\left(\mathbf{v} ; \mathbf{v}_{1}\right)\right) \subset B\left(\mathbf{v}_{1}, r_{\max }\right),
$$

where $\operatorname{supp}(f)$ is the support of $f, B\left(\mathbf{v}_{1}, r_{\max }\right)$ is a ball centered at $\mathbf{v}_{1}$ with radius $r_{\text {max }}$. In addition to the two observations described above, we also find that many particles, especially negative ones, are quite close to $\mathbf{u}_{m}$, so that a more efficient choice of $r_{\max }$ would be to make it $\mathbf{v}_{1}$-dependent. One could precompute the relation between the support size and the distance $\left|\mathbf{v}_{1}-\mathbf{u}_{m}\right|$ and assign each source particle a different $r_{\max }$. This could greatly accelerate the computation and will be studied in a future work. 


\section{Summary of Algorithm and Acceleration technique}

We summarize the whole algorithm in this section for general bilinear collision operators, including those for both RGD and Coulomb collisions.

Initialization: Choose the effective number $N_{\text {eff }}$. For the initial distribution

$$
f^{I}=m^{I}-f_{n}^{I}+f_{p}^{I}
$$

sample $N_{p}=\frac{\rho_{p}^{I}}{N_{\text {eff }}}$ P-particles from $f_{p}^{I}, N_{n}=\frac{\rho_{n}^{I}}{N_{\text {eff }}}$ N-particles from $f_{n}^{I}$ and $N_{\tilde{f}}=\alpha\left(N_{p}+N_{n}\right)$ F-particles from $f^{I}$. Here $\alpha \gtrsim 1$ is a constant. In our simulations we find $\alpha=1.2$ gives quite satisfactory efficiency.

One step: Suppose at time $t$, we have $N_{p}$ P-particles, $N_{n}$ N-particles and $N_{\tilde{f}} \geq\left(N_{p}+N_{n}\right)$ F-particles, as well as (the moments of) the Maxwellian component $m(t)$. Then

- Step 1. Perform F-P and F-N collisions.

- Choose $N_{p}$ F-particles and calculate collisions between these F-Particles and the Pparticles. Collect the post-collisional P-particles.

- Choose another $N_{n}$ F-particles and calculate collisions between these F-Particles and the N-particles. Collect the post-collisional N-particles.

- Step 2. Sample from $\Delta t\left(Q\left(f_{p}-f_{n}\right), m\right)$.

- A total number $\mathcal{O}\left(\Delta t\left(N_{p}+N_{n}\right)\right)$ of additional $\mathrm{P}$ and $\mathrm{N}$ particles are sampled from $\Delta t\left(Q\left(f_{p}-f_{n}\right), m\right)$, as described in Section 4.2.2. In this step one still uses the $\mathrm{P}$ and $\mathrm{N}$ particles before Step 1 to represent $f_{p}$ and $f_{n}$ in the sampling calculations.

- Step 3. Update $\mathrm{P}$ and $\mathrm{N}$ particles.

- The post-collisional particles collected in Step 1 and the P particles sampled from Step 2 give the P-particles at time $(t+\Delta t)$.

- The post-collisional particles collected in Step 1 and the N particles sampled from Step 2 give the $\mathrm{N}$-particles at time $(t+\Delta t)$.

- Update $N_{p}$ and $N_{n}$ accordingly.

- Step 4. Enforce conservation.

- Conserve the total density by adjusting $\rho_{m}$, the density of the Maxwellian part.

- Conserve the total momentum by shifting the P-particles and N-particles.

- Conserve the total energy by rescaling the P-particles and N-particles.

- Step 5. Perform F-F collisions.

- A total number of $N_{\tilde{f}} / 2$ collisions are performed for all the F-particles. The collection of post-collisional particles give the F-particles at time $(t+\Delta t)$.

- Step 6. Particle resampling.

- Use the algorithm described in Section 3.5 to reduce the particle numbers, if condition (3.3) is violated. 


\section{Numerical example}

In the following we perform numerical tests for Coulomb collisions in spatially homogenous systems with three dimensional velocities. We apply the TA method on the collision part, with the constant coefficient $A=1$ in (2.8). We use the name "negative TA method" for our method, and "regular TA method" for the original TA method [21].

In the particle resampling method described in Section 3.5, we take the cut off to be $K=10$. Unless specified, we take the initial ratio of $N_{\tilde{f}}$ over $N_{p}+N_{n}$ to be $\alpha=1.2$, i.e. the particle resampling is performed when $\left(N_{p}+N_{n}\right)$ grows by $20 \%$. Moreover, we require the number of Fparticles to be above a minimum value. In simulations we take $N_{\tilde{f}} \geq 5000$. The time step is always $\Delta t=10^{-3}$.

In this work we always take the Maxwellian part $m(\mathbf{v})$ to be the global Maxwellian, except for small variation in density $\rho_{m}$ due to density conservation step. So we have $N_{p} \approx N_{n}$ for all the time. The time variant $m(\mathbf{v})$ will be investigated in future work.

All the simulations are based on three examples.

Two-Temperature Maxwellian relaxation. The initial data for this examle is

$$
f^{I}(\mathbf{v})=\frac{\rho}{(2 \pi)^{3 / 2} T \sqrt{T+\delta T}} \exp \left(-\frac{v_{x}^{2}+v_{y}^{2}}{2 T}\right) \exp \left(-\frac{v_{z}^{2}}{2(T+\delta T)}\right) .
$$

The temperature difference $\delta T$ relaxes to 0 as time evolves. We want to show our method is applicable to all regimes, so initially we take

$$
\rho=1, \quad T=1, \quad \delta T=1,
$$

which is a large deviation from equilibrium.

Bump-on-Tail problem. The initial value for the Bump-on-Tail problem consists of a central Maxwellian and a small bump at high energy,

$$
f^{I}(\mathbf{v})=\frac{\beta \rho}{(2 \pi T)^{3 / 2}} \exp \left(-\frac{|\mathbf{v}|^{2}}{2 T}\right)+\frac{(1-\beta) \rho}{\left(2 \pi T_{b}\right)^{3 / 2}} \exp \left(-\frac{\left|\mathbf{v}-\mathbf{u}_{b}\right|^{2}}{2 T_{b}}\right)
$$

where

$$
\rho=1, \quad \beta=0.9, \quad T=1, \quad T_{b}=0.01, \quad \mathbf{u}_{b}=[5,0,0] .
$$

This problem has been studied extensively for the TD method $[6,17]$.

Rosenbluth's test problem. This test starts with volcano-like initial data [18],

$$
f^{I}(\mathbf{v})=0.01 \exp \left(-10(|\mathbf{v}|-1)^{2}\right) .
$$

In this test the distribution $f$ stays radially symmetric for all time, making it easier to recover the 1D distribution $|\mathbf{v}|^{2} f(|\mathbf{v}|)$ by counting the number of particles within each energy interval. Hence we use this test to check the entropy evolution and the numerical error in the $L^{1}$ norm.

\subsection{Conservation of moments}

In the first test we check the time evolution of the moments, without enforcing the moment conservation (the Step 4 in Section 5),for Rosenbluth's test problem. Figure 1 shows the time evolution of the relative change in moments: (top) the density $(\rho(t)-\rho(0)) / \rho(0)$, (middle) the first component of momentum $\rho(t) \mathbf{u}_{1}(t)$ and (bottom) the energy $(E(t)-E(0)) / E(0)$. The other two components of momentum are similar to $\rho(t) \mathbf{u}_{1}(t)$ and not shown. Several realizations are shown in each figure. The moments are conserved up to the statistical error.

In the following sections we always enforce the moment conservation. 

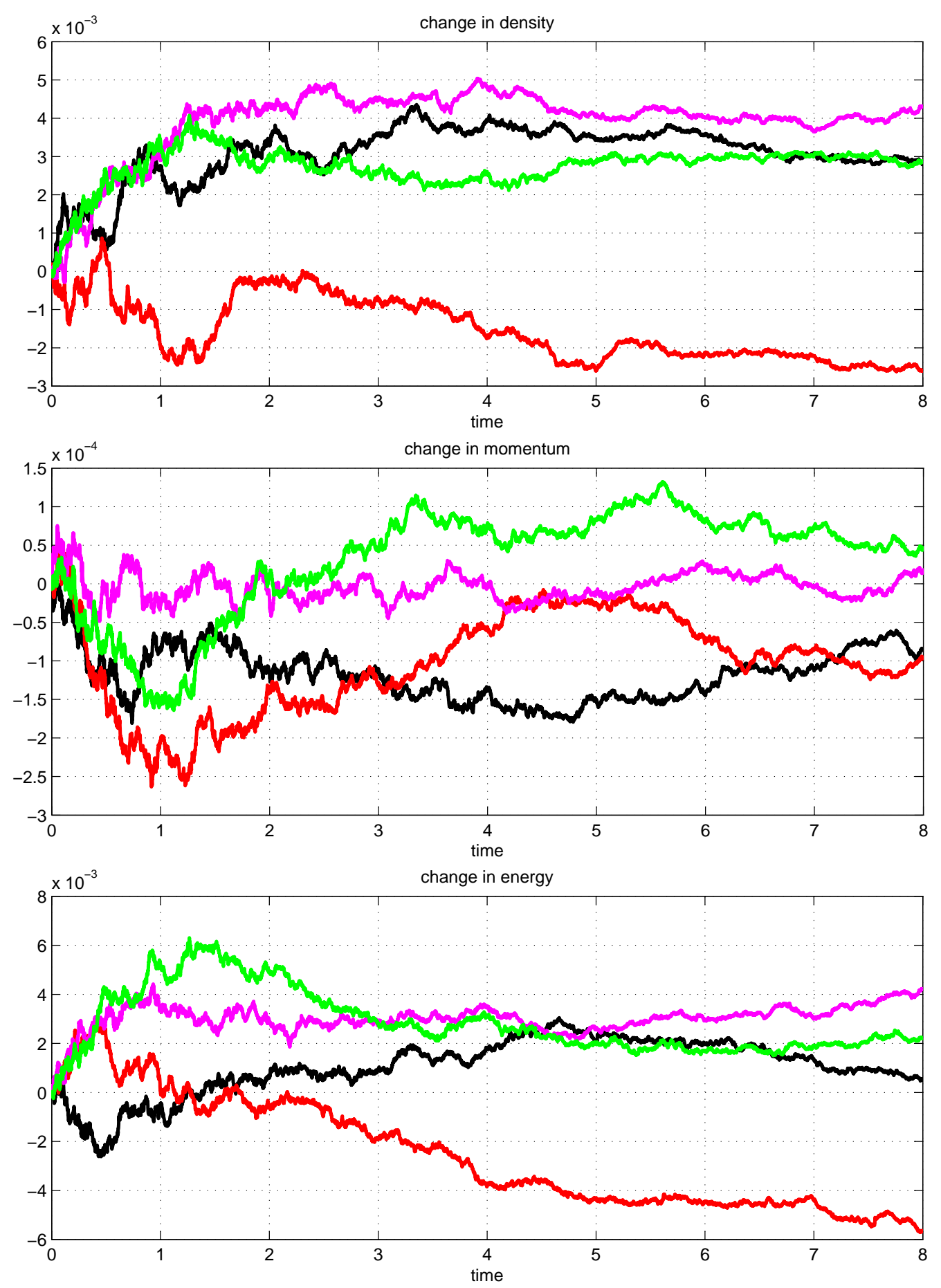

Figure 1: The time evolution of the relative change in moments: (top) the density $(\rho(t)-\rho(0)) / \rho(0)$, (middle) the first component of momentum $\rho(t) \mathbf{u}_{1}(t)$ and (bottom) the energy $(E(t)-E(0)) / E(0)$. The test is based on Rosenbluth's problem with initially $N_{p}=40,000$. Four realizations are included. In this test the moment conservation step is not applied. 


\subsection{Particle Resampling}

In this test we numerically check the particle resampling method described in Section 3.5. We apply Rosenbluth's test problem since its distribution is radially symmetric and hence easy to show.

Figure 2 shows the distribution of $r^{2} f_{p}(r)$ and $r^{2} f_{n}(r)$ before (top) and after (middle) the first application of particle resampling method, here $r=|\mathbf{v}|$. It clearly shows that after cancellation there is no overlap in the supports of $f_{p}$ and $f_{n}$. Note that we are performing the particle resampling in the fully 3D space. Therefore a very small overlap can still be observed after these particles are projected to $1 \mathrm{D}$ energy space.

The bottom of Figure 2 plots the distributions $r^{2}\left(f_{p}-f_{n}\right)$ before and after the resampling, showing that the resampling does not change the total distribution.

\subsection{Two-Temperature Maxwellian}

Next we apply the negative particle method to the Two-Temperature Maxwellian problem.

Figure 3 shows the time evolution of the numbers of $\mathrm{P}$ (blue dashed line), N (red dotted line) and $\mathrm{F}$ (green solid line) particles. Here we take $\alpha=1.1$. The lines for $\mathrm{P}$ and $\mathrm{N}$ particles are almost identical. The green line shows the evolution of $\frac{N_{\tilde{f}}}{2}$. We start with $N_{p}=N_{n}=40,000$ and $N_{\tilde{f}}=88,000$. The particle resampling is performed when $\left(N_{p}+N_{n}\right)>N_{\tilde{f}}$. After each resampling $N_{p}$ and $N_{n}$ jump to much lower value since both distributions are approaching the same equilibrium. $N_{\tilde{f}}$ is also reduced to save computational cost since we only need $N_{\tilde{f}}=\alpha\left(N_{p}+N_{n}\right)$. Eventually the minimum value $N_{\tilde{f}}=5000$ is reached.

The time evolution of $\delta T / \delta T(0)$ is shown in Figure 4, in which the deviation of temperature is defined by $\delta T=\int\left(v_{z}^{2}-\frac{1}{2}\left(v_{x}^{2}+v_{y}^{2}\right)\right) f(\mathbf{v}) \mathrm{d} \mathbf{v}$. The blue solid line is solved by a regular TA method with $10^{6}$ particles as a reference. The red dotted line is the negative particle method with initially $N_{p}=N_{n}=40,000$. This line overlaps the first line, showing that our method computes the time evolution of $\delta T / \delta T(0)$ accurately, although very fewer particles are used. The green dashed line gives the $\delta T / \delta T(0)$ computed from the F-particles. As time evolves the statistical error becomes more obvious, due to the fact that the number of F-particles is reduced (see Figure 3). Nonetheless, the finer solution based on these F-particles still gives very accurate result.

\subsection{Bump on Tail problem}

In this section we apply the negative particle method to the Bump on Tail problem.

Figure 5 shows the snapshots of the marginal distribution

$$
f\left(v_{x}\right)=\int f\left(v_{x}, v_{y}, v_{z}\right) \mathrm{d} v_{y} \mathrm{~d} v_{z}
$$

at different times. The value of the one dimensional distribution $f_{p}\left(v_{x}\right)$ is recovered by counting the number of $\mathrm{P}$ particles whose first coordinate falls in a prescribed bin $\left[v_{x}-\Delta v / 2, v_{x}+\Delta v / 2\right]$. The recovering of $f_{n}$ is similar.

The blue solid line comes from the negative particle method with initially $N_{p}=N_{n}=40,000$ particles; the red dashed line corresponds to the regular TA method with $10^{6}$ particles as a reference. Both solutions are approaching equilibrium and agree very well. Figure 6 shows the snapshots of the three components $m, f_{p}$ and $f_{n}$ at various times. The Maxwellian component $m$ (black dotted line) is time invariant. The high energy portion of the distribution $f_{p}$ (red dashed line) decays and moves toward the global Maxwellian. The distribution $f_{n}$ (blue solid line) also decays. Note that $f_{n}$ is always below $m$, so that the whole distribution $f=m+f_{p}-f_{n}$ stays positive. The fact that $f_{p}$ and $f_{n}$ decay in time indicates that fewer $\mathrm{P}$ and $\mathrm{N}$ particles are used as time evolves. 

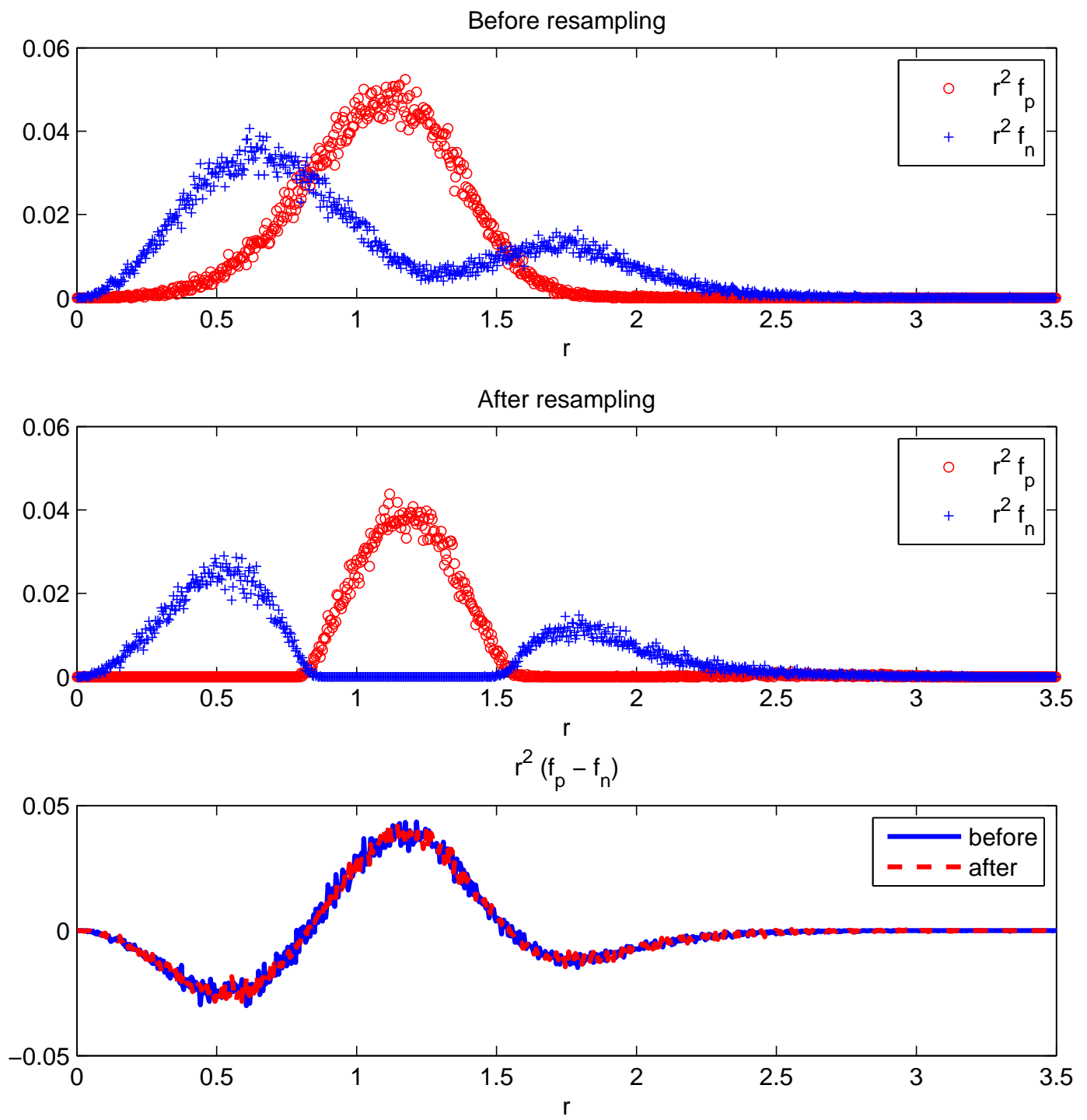

Figure 2: Particle resampling in Rosenbluth's test problem. Top: the distribution $r^{2} f_{p}$ (red circle) and $r^{2} f_{n}$ (blue + ) before the resampling. Middle: the distribution $r^{2} f_{p}$ (red circle) and $r^{2} f_{n}$ (blue + ) after the resampling. Bottom: the distribution $r^{2}\left(f_{p}-f_{n}\right)$ before (blue solid line) and after (red dashed line) the resampling. 


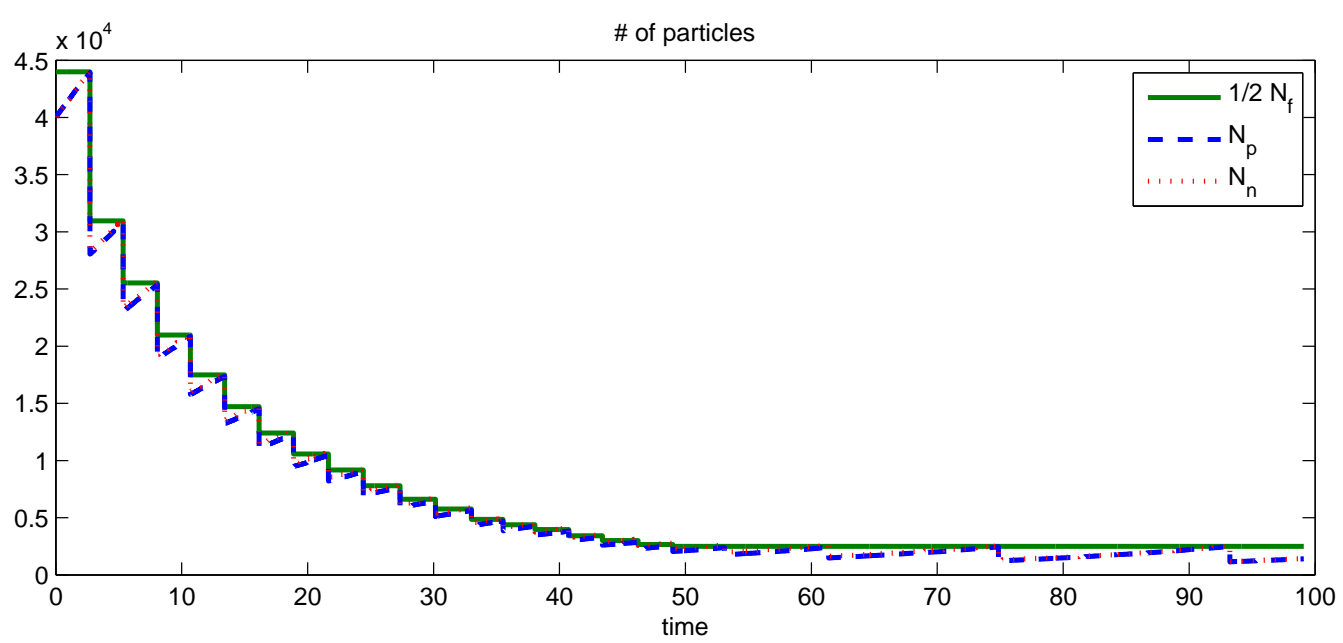

Figure 3: The number of the particles vs. time, for the Two-Temperature Maxwellian problem. The sudden jumps correspond to applications of particle resampling.

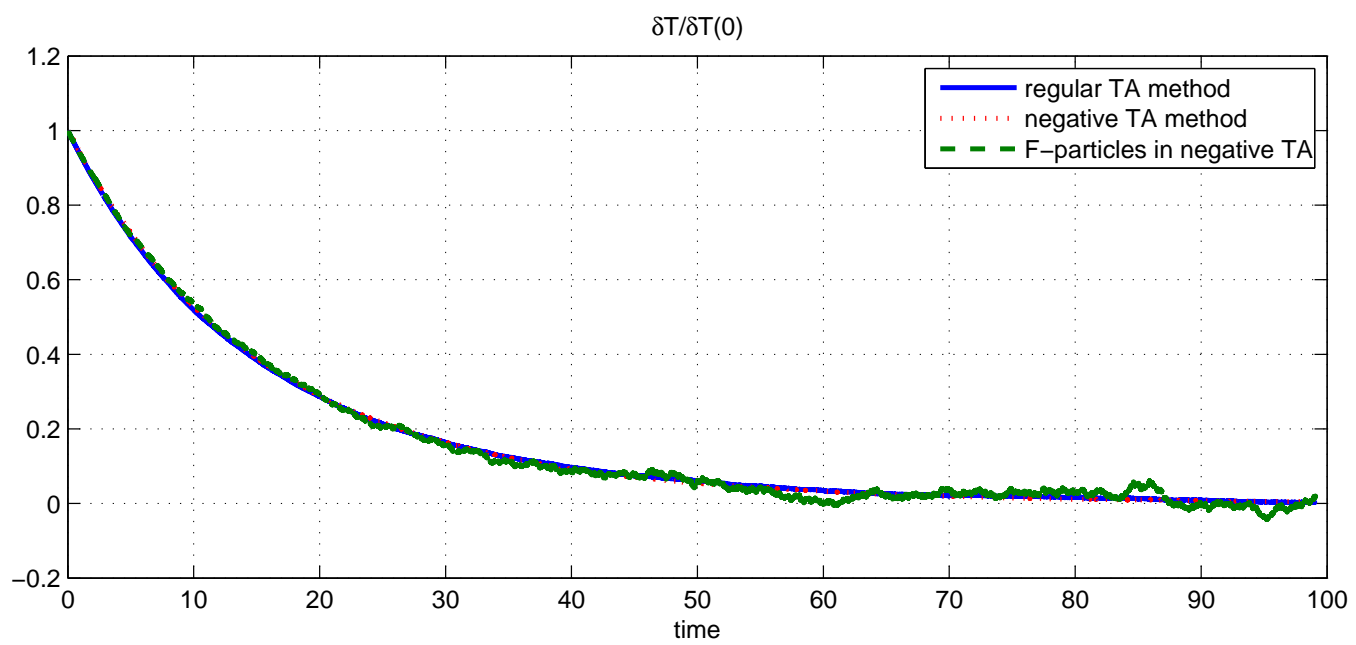

Figure 4: Time evolution of $\delta T / \delta T(0)$ in the Two-Temperature Maxwellian problem, obtained from a regular TA method with $10^{6}$ particles (blue solid line); a negative particle method with initial values $N_{p}=N_{n}=40,000$ (red dotted line); the F-particles in the negative particle method (green dashed line). The first two lines are nearly identical. 

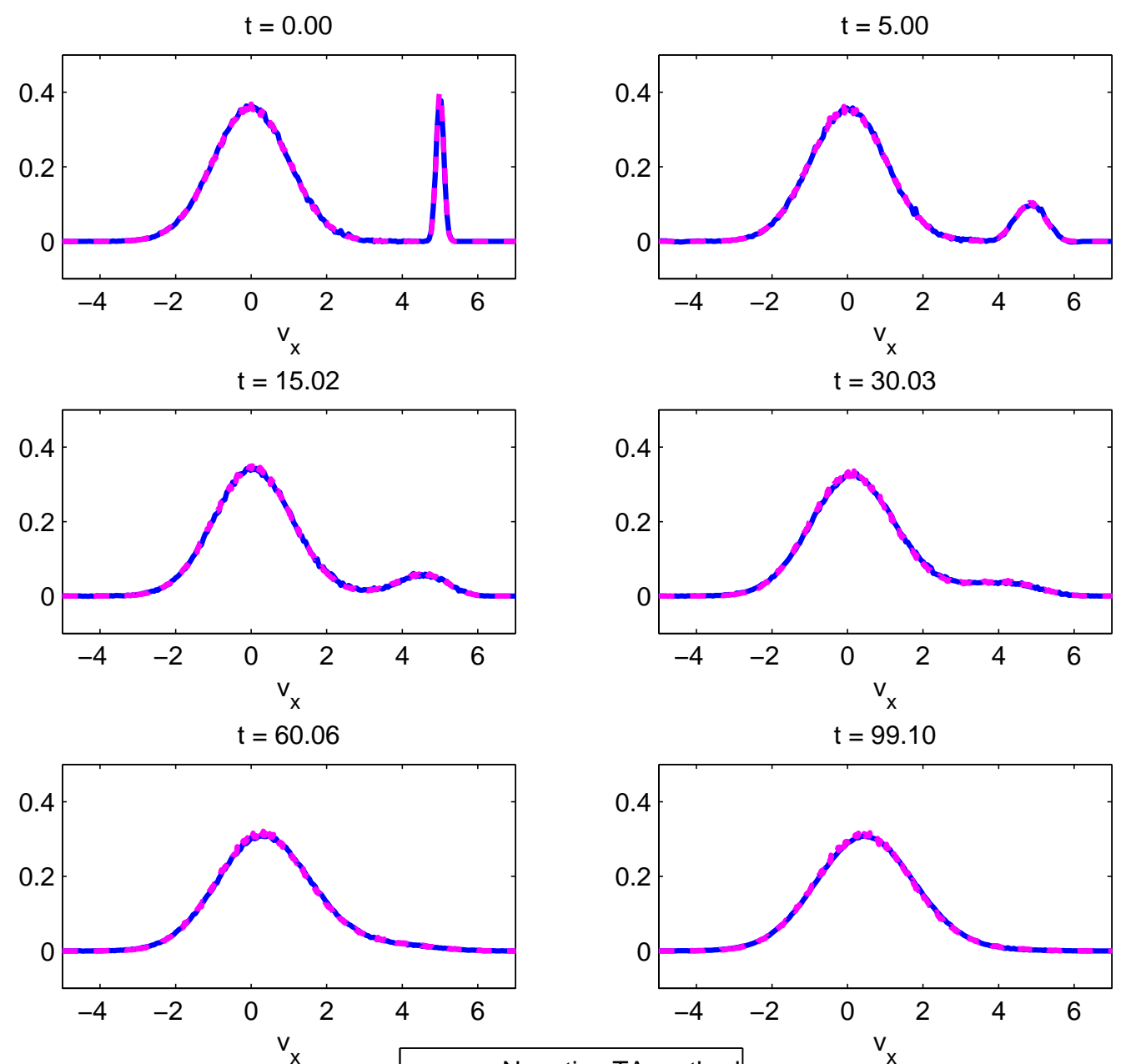

Negative TA method

- - - Regular TA method

Figure 5: Snapshots at various times of the marginal distribution $\int f\left(v_{x}, v_{y}, v_{z}\right) \mathrm{d} v_{y} \mathrm{~d} v_{z}$ in the Bump-on-Tail problem. Red dashed line: regular TA method with $10^{6}$ particles. Blue solid line: negative particle method with $N_{p}=40,000$ initially.

The $\mathrm{P}$ and $\mathrm{N}$ particles in the central part of frepresent the difference between the central Maxwellian and the global Maxwellian, and their decay is slow. If one were to take the central Maxwellian as $m$ in the splitting (2.1) and evolves $m$ correctly, one would expect to see the central particles get damped quickly, leaving the high energy tail slowly moving to the central Maxwellian. This will be investigated in a future work.

\subsection{Rosenbluth's problem}

In this section we apply the negative particle method to Rosenbluth's test problem.

Figure 7 shows snapshots of the distribution

$$
r^{2} f(r)=\int_{|\mathbf{v}|=r} f(\mathbf{v}) \mathrm{d} \mathbf{v}
$$

at various times. The value of the one dimensional distribution $r^{2} f_{p}(r)\left(r^{2} f_{n}(r)\right)$ is recovered by 

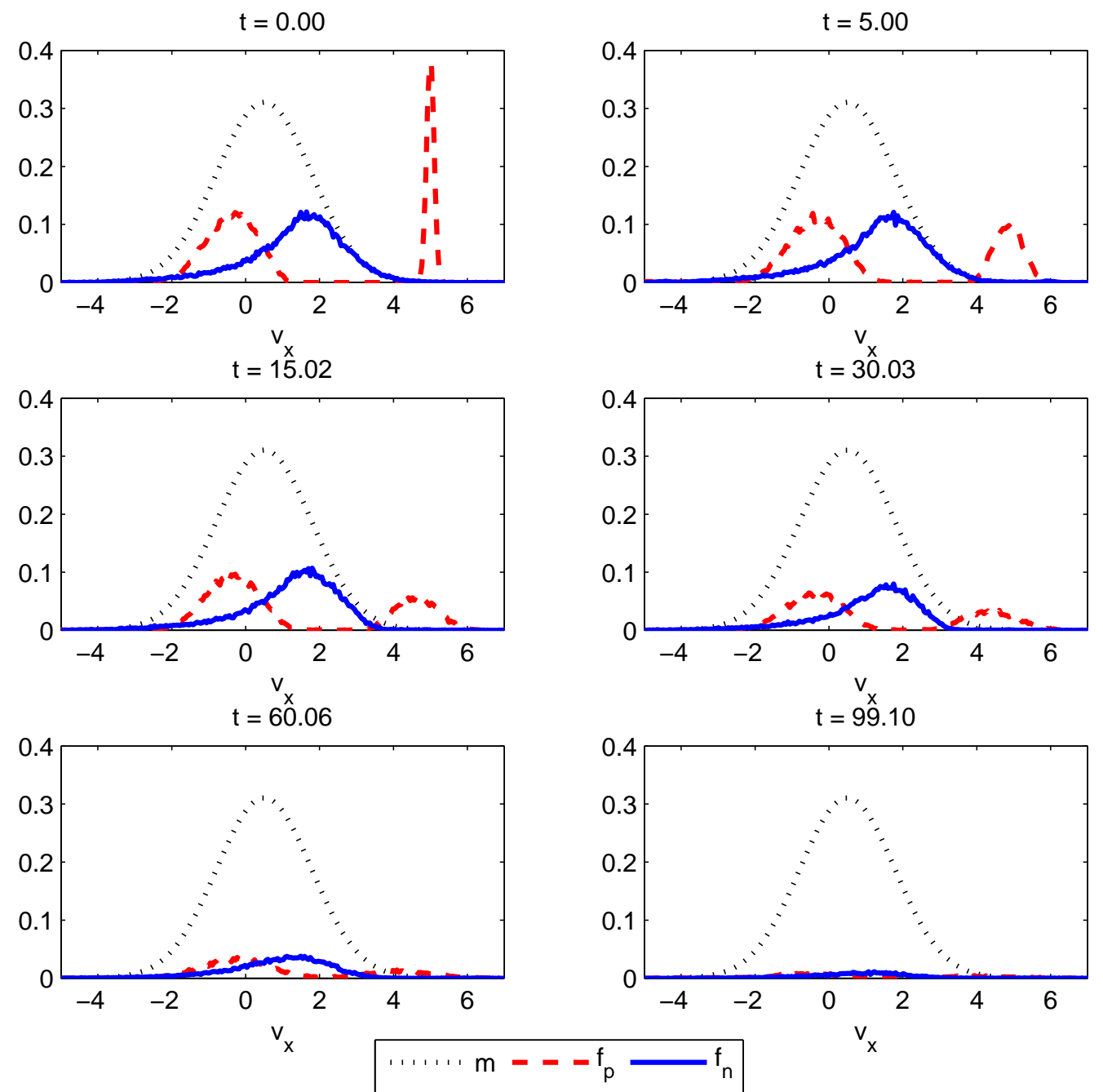

Figure 6: Snapshots at various times of the components $m, f_{p}$ and $f_{n}$ in the Bump-on-Tail problem. 
counting the number of $\mathrm{P}(\mathrm{N})$ particles whose (square root of) energy $|\mathbf{v}|$ falls in a prescribed bin $[r-\Delta r / 2, r+\Delta r / 2]$.

The blue solid line corresponds to the negative particle method with initially $N_{p}=N_{n}=40,000$ particles; the red dashed line corresponds to the regular TA method with $10^{6}$ particles. As in the Two-Temperature Maxwellian test, both solutions are approaching equilibrium and agree well. Figure 8 shows snapshots of the three components $r^{2} m(r), r^{2} f_{p}(r)$ and $r^{2} f_{n}(r)$ at various times. Decay of $r^{2} f_{p}(r)$ and $r^{2} f_{n}(r)$ indicates that the number of $\mathrm{P}$ and $\mathrm{N}$ particles are reduced.

Figure 9 shows the time evolution of entropy defined by

$$
H=\int f(\mathbf{v}) \log \frac{f(\mathbf{v})}{M(\mathbf{v})} \mathrm{d} \mathbf{v}=4 \pi \int r^{2} f(r) \log \frac{f(r)}{M(r)} \mathrm{d} r
$$

with $M(\mathbf{v})$ the global Maxwellian. $H(t) / H(0)$ is plotted. The red dashed line corresponds to the negative particle method while the reference solution (blue solid line) comes from the regular TA method with $10^{6}$ particles. The two lines agree quite well.

\subsection{Convergence test}

Now we perform a numerical test to check convergence of the negative particle method, as particle number $N_{p}, N_{n} \rightarrow \infty$. The test is based on the Rosenbluth's problem since it has a one dimensional solution. we consider the time-averaged error in $L^{1}$ norm,

$$
\operatorname{Error}(t)=\frac{1}{t} \int_{0}^{t}\left\|f(\tau, \mathbf{v})-f_{\operatorname{ref}}(\tau, \mathbf{v})\right\|_{1} \mathrm{~d} \tau=\frac{4 \pi}{t} \int_{0}^{t} \mathrm{~d} \tau \int\left|r^{2} f(\tau, r)-r^{2} f_{\operatorname{ref}}(\tau, r)\right| \mathrm{d} r
$$

where $f_{\text {ref }}(\mathbf{v})$ is a reference solution solved by the regular TA method with $10^{7}$ particles.

The top of Figure 10 shows the evolution of particle numbers in this test with initially $N_{p}=$ 40,000 to illustrate the time when particle resampling is performed. The time of resampling is approximately the same for other initial values of $N_{p}$.

The middle of Figure 10 shows the time evolution of $\left\|f(t, \mathbf{v})-f_{\text {ref }}(t, \mathbf{v})\right\|_{1}$ and $\| \tilde{f}(t, \mathbf{v})-$ $f_{\text {ref }}(t, \mathbf{v}) \|_{1}$, in which $f$ comes from the negative particle method with initially $N_{p}=N_{n}=40,000$, while $\tilde{f}$ is the coarse solution represented by F-particles. Initially the number of F-particle is $N_{\tilde{f}}=96,000$. As illustrated in the figure, initially the coarse solution $\tilde{f}$ gives a slightly better solution than $f$, since the initial distribution is far away from the equilibrium and there are a relatively large number of F-particles. However as time evolves the error in $\tilde{f}$ is growing since the number of F-particles is decreasing (see the green solid line in the top of Figure 10). The error in $f$ is decreasing because the statistical error is given by

$$
\frac{\rho_{p}}{\sqrt{N_{p}}}=\sqrt{\rho_{p} N_{\mathrm{eff}}}
$$

and as time evolves the density $\rho_{p}$ decreases, while the effective number $N_{\text {eff }}$ is constant.

The bottom of Figure 10 shows the accumulated error (6.2) at different times $t$. We choose three different ending times. (a) $t=0.4$ when there is no particle resampling performed; (b) $t=2$ when the resampling has been performed several times but the system has not reached equilibrium yet; (c) $t=9$ when the system is very close to equilibrium. This figure shows the results of the error from both the coarse solution $\tilde{f}$ and the fine solution $f$. The negative particle method is solved with initial $N_{p}=10,000,20,000,40,000,80,000,160,000$. For comparison the figure also shows a line with slope $-1 / 2$. Clearly all these error exhibit a half order convergence, as initial $N_{p} \rightarrow \infty$. The figure does not include results for the coarse solution $\tilde{f}$ at time $t=9$, since for all these tests, the number of F-particles reaches the minimum value $N_{\tilde{f}}=5000$ at $t=9$. 

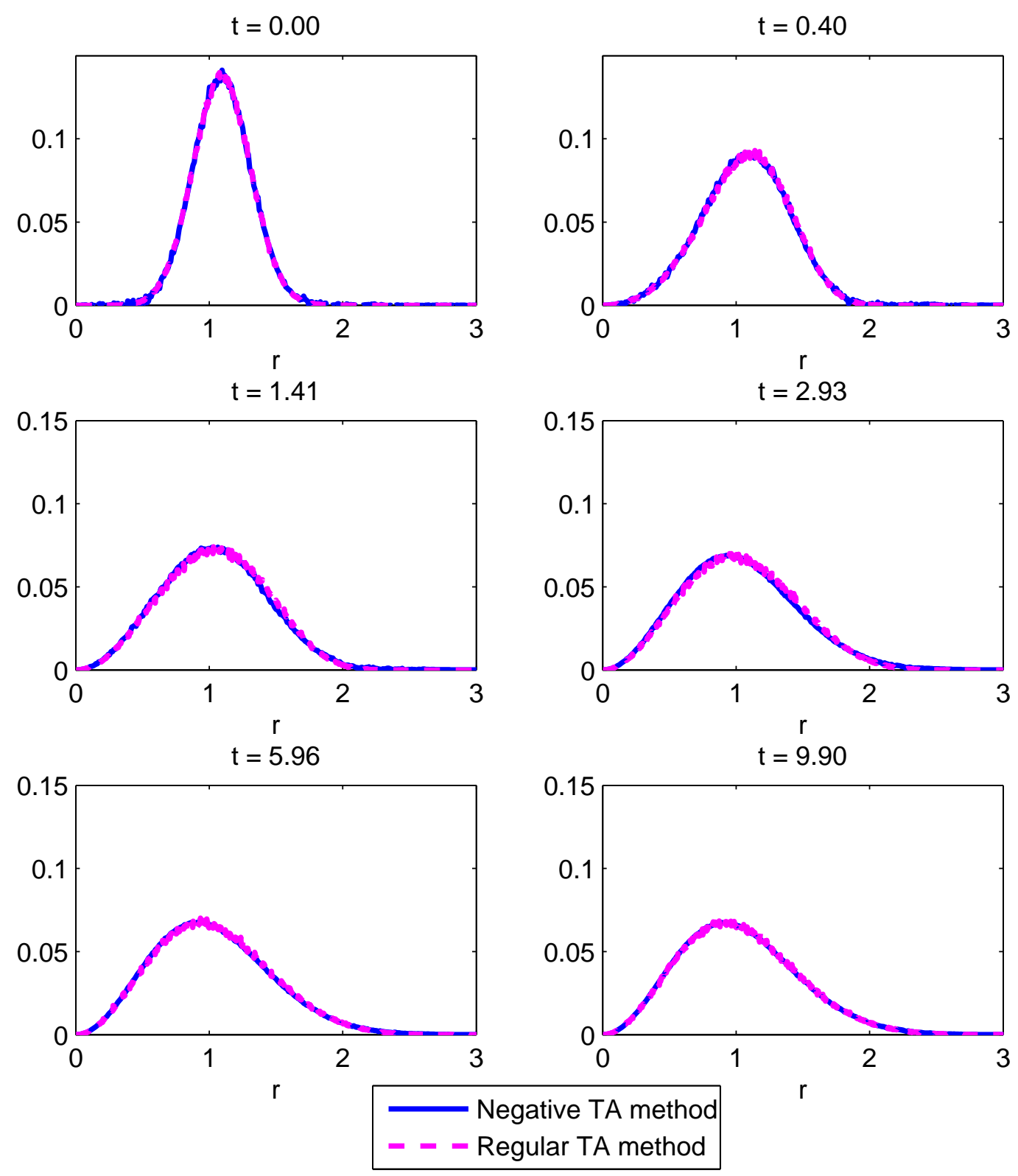

Figure 7: Snapshots at various times of the radial symmetric distribution $r^{2} f(r)$ in Rosenbluth's test problem. Red dashed line: regular TA method with $10^{6}$ particles. Blue solid line: negative particle method with $N_{p}=40,000$ initially. 

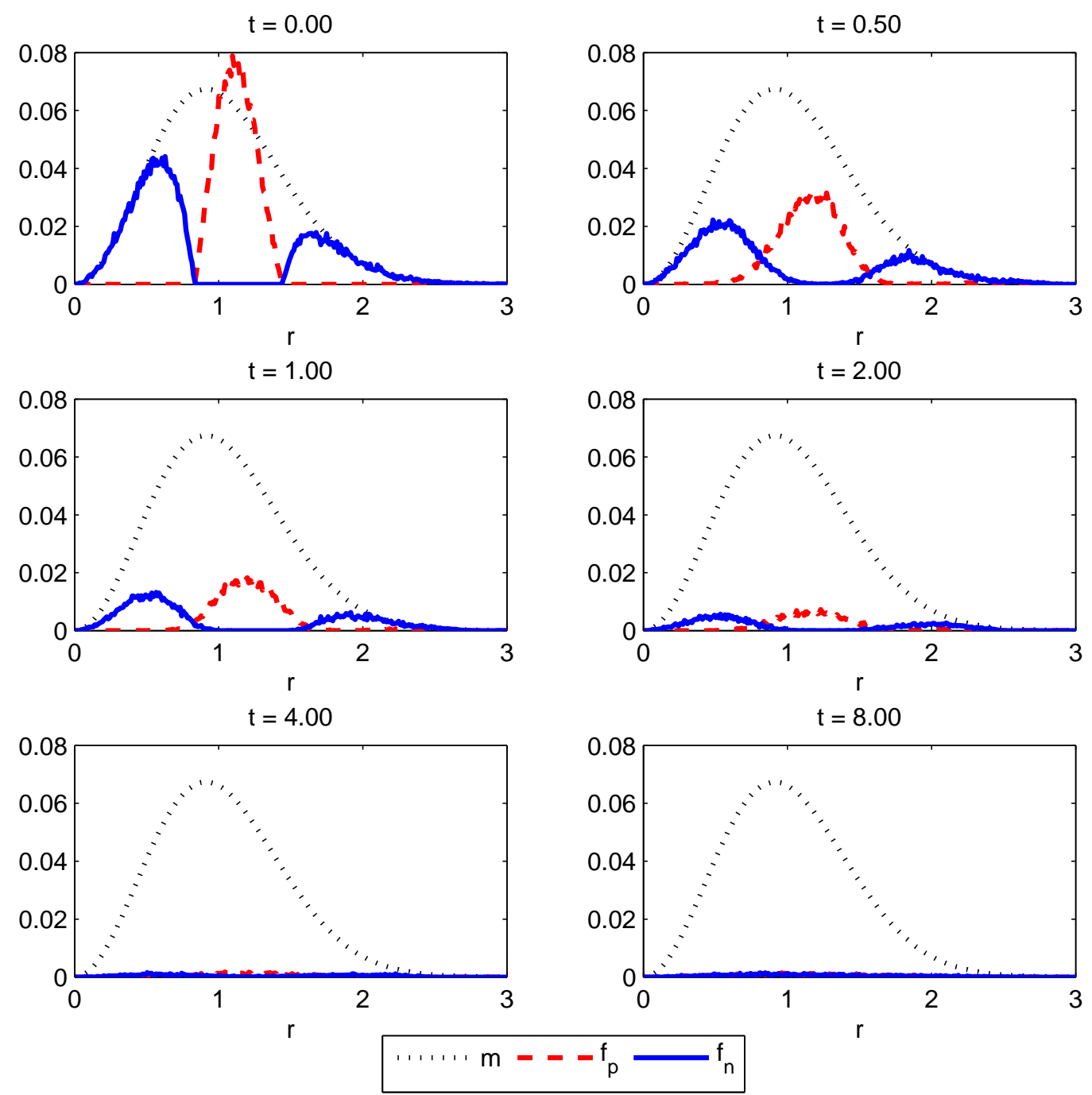

Figure 8: Snapshots at various times of the components $r^{2} m, r^{2} f_{p}$ and $r^{2} f_{n}$ in Rosenbluth's test problem.

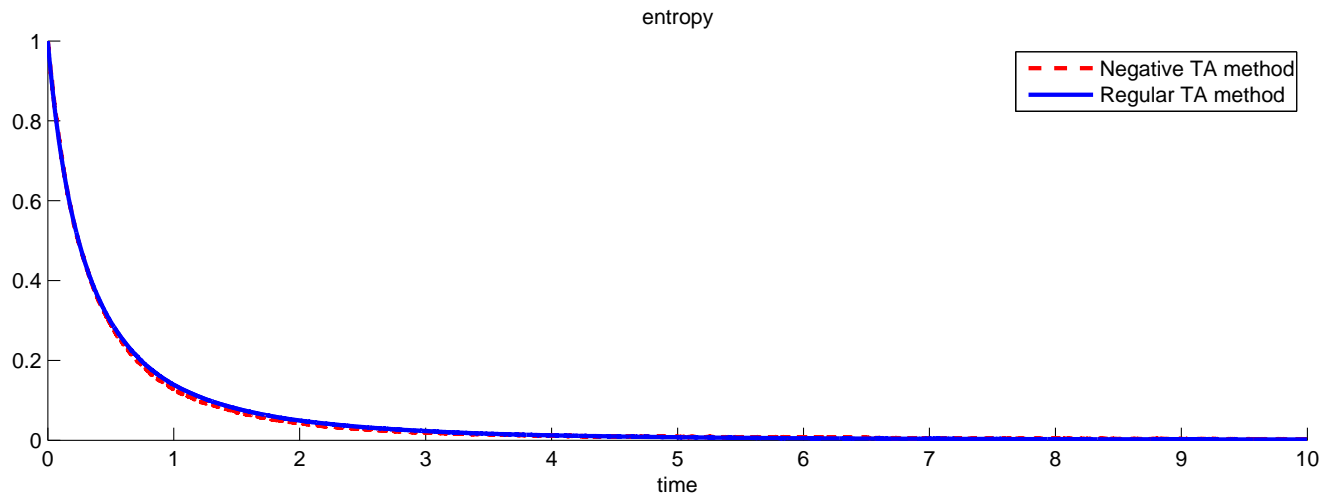

Figure 9: Time evolution of entropy $H(t) / H(0)$ in Rosenbluth's test problem. Blue solid line: regular TA method with $10^{6}$ particles. Red dashed line: negative particle method with $N_{p}=40,000$ initially. 

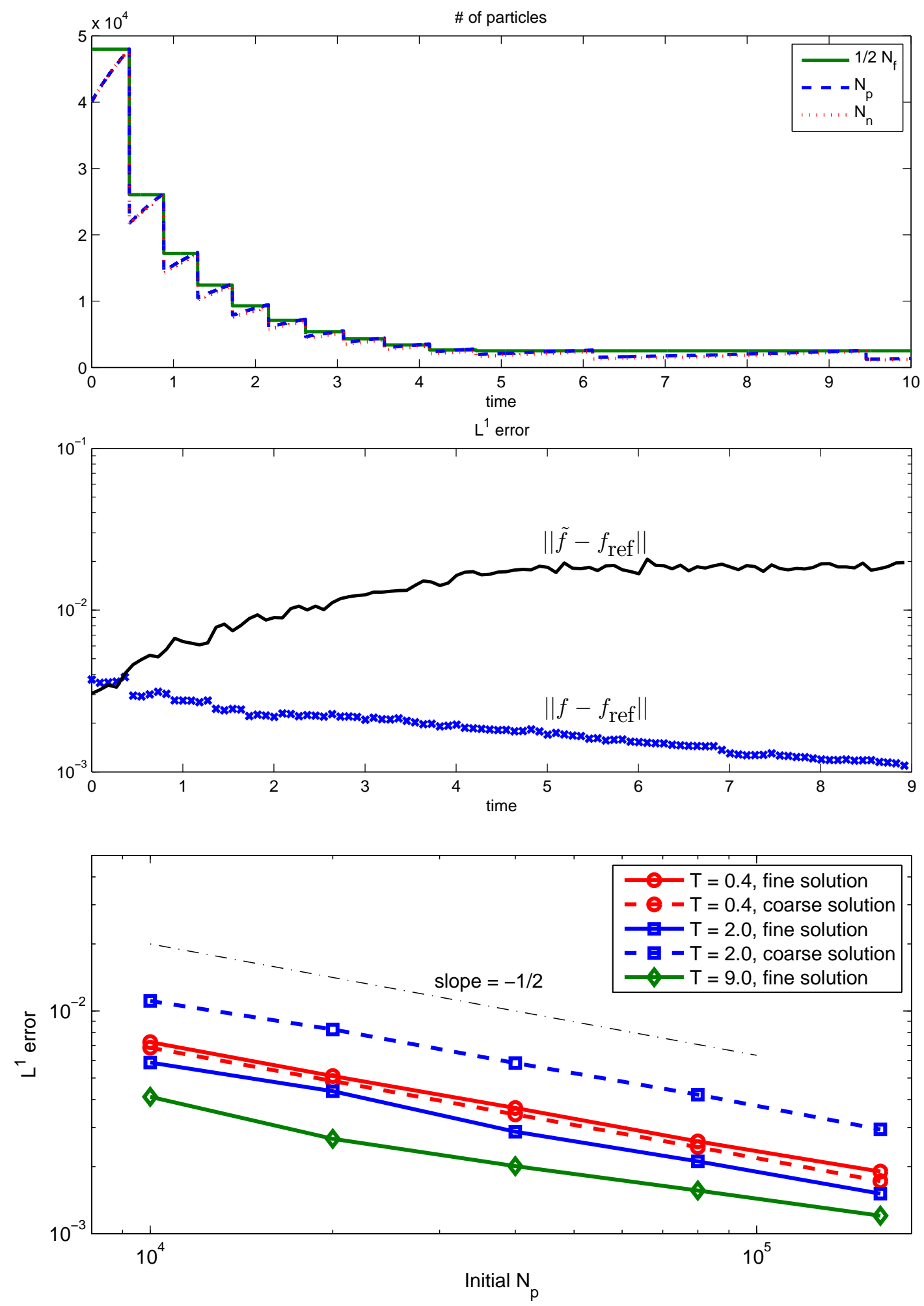

Figure 10: The convergence test on Rosenbluth's test problem. Top: evolution of the numbers of particles. Middle: time evolution of error in $L^{1}$ norm. Bottom: error vs $N_{p}(0)$ for fine solution $f$ and coarse solution $\tilde{f}$ at different times. 

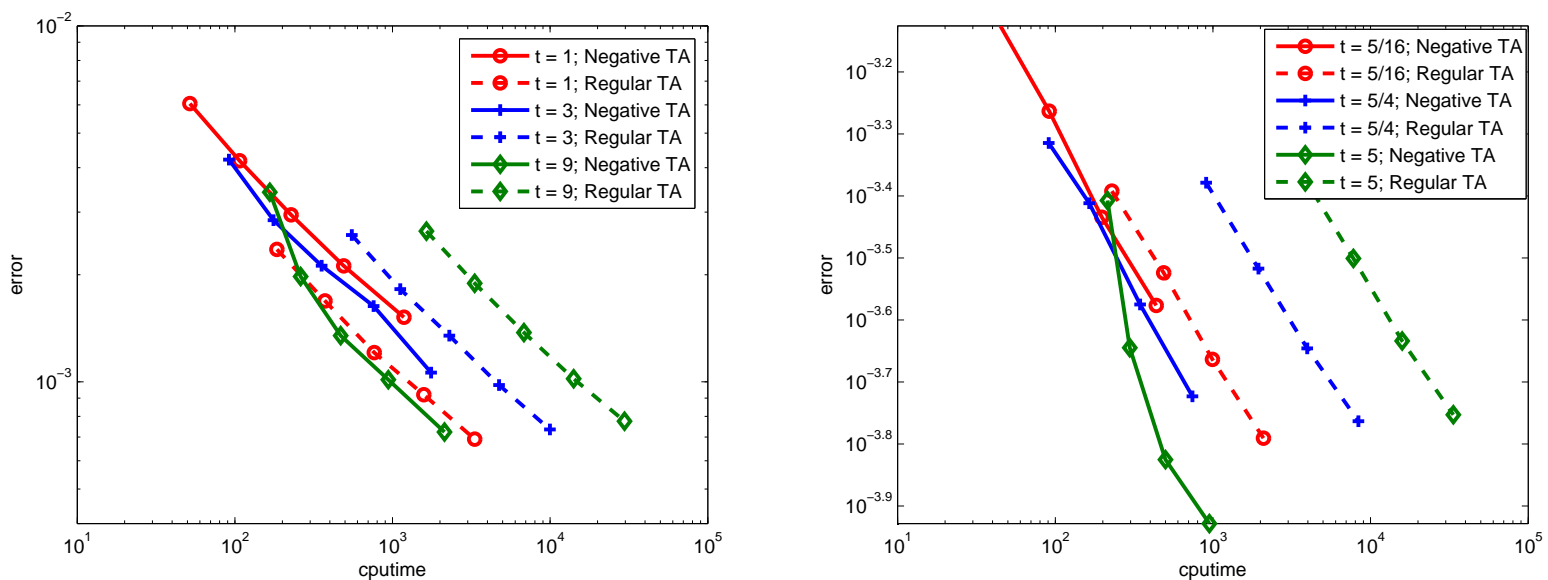

Figure 11: The efficiency test on Rosenbluth's test problem. Left: start with (6.1); Right: start with (6.3).

\subsection{Efficiency test}

Finally we check the efficiency of our negative particle method. The negative particle method is designed to be efficient when the distribution $f$ is close to the equilibrium $m$. We define the distance between $f$ and $m$ in $L^{1}$ norm as

$$
\Gamma(t)=\frac{\|f(t)-m(t)\|_{1}}{\|f(0)\|_{1}}
$$

and check the performance of our method with different $\Gamma$.

We record the cpu time used in a simulation up to time $t$ and compute the time averaged error (6.2) during the time period $[0, t]$. In the left of Figure 11, we plot the relation between the cpu time and the error up to time $t=1$ (red circles), $t=3$ (blue + ) and $t=9$ (green diamonds), based on the Rosenbluth's test problem (6.1). The results correspond to the negative particle method are computed with initially $N_{p}=10^{4}, 2 \times 10^{4}, 4 \times 10^{4}, 8 \times 10^{4}, 1.6 \times 10^{5}$, and shown in solid lines; while the results correspond to the regular TA method are computed with $N=5 \times 10^{5}, 10^{6}, 2 \times 10^{6}, 4 \times 10^{6}, 8 \times 10^{6}$, and shown in dashed lines. The error is computed by comparing to a reference solution solved by regular TA method with $N=10^{7}$.

In this case the initial distribution is quite far away from equilibrium, with $\Gamma(0) \approx 0.7$. At the three times $t=1,3,9, \Gamma(t) / \Gamma(0)=0.4,0.1,0.01$ (respectively). The figure shows that until time $t=1$ the regular TA method is more efficient than the negative particle method. But as time evolves the negative particle method becomes more efficient as the distribution approaches equilibrium. At the final time $t=9$, the negative particle method is about 20 times faster than the regular method.

The right side of Figure 11 shows the corresponding result for initial data closer to equilibrium,

$$
f^{I}(\mathbf{v})=0.01 \exp \left(-10(|\mathbf{v}|-0.5)^{2}\right)
$$

In this case $\Gamma(0)=0.36$. The results are shown in the right of Figure 11, with ending times $t=5 / 16$ (red circles), $t=5 / 4$ (blue + ) and $t=5$ (green diamonds), corresponding to $\Gamma(t) / \Gamma(0)=$ $0.37,0.07,0.007$ respectively. Again the results for the negative particle method are shown as solid lines while those from regular TA method are shown in dashed lines. Note that the negative particle method is about 100 times faster than the regular method with the ending time $t=5$. 


\section{Conclusions and Future Work}

In this work we develop a new negative particle method for the general bilinear collision operators in the spatial homogeneous case and apply it to Coulomb collisions. We introduce the use of Fparticles, which coarsely and independently approximate the whole distribution. With the aid of F-particles an efficient negative particle method is designed based on a new operator decomposition. This new method successfully reduces the growth of particle numbers from the numerical scale to the physical scale for Coulomb collisions. We also propose a particle resampling method to reduce the particle number and further improve the efficiency. Various numerical simulations are performed to demonstrate the accuracy and efficiency of this method.

There are several aspects deserve future investigation:

- In this work the Maxwellian part $m$ is fixed. It may be possible to further reduce the number of particles by evolving $m$, for example as in [12].

- The combination with the negative particle method with the thermalization/dethermalization method [17] seems to be promising since the thermalization process reduces the number of particle while maintaining first order accuracy in the time discretization.

- This method is applicable to general collision operators. However for each operator one needs to design a suitable method to sample from the source term $\Delta t Q\left(f_{p}-f_{n}, m\right)$. This needs to be studied case by case.

- The extension to spatial inhomogeneous problems will be investigated in future work.

\section{Acknowledgement}

This work started in discussions with Prof. Antoine Cerfon from New York University and Dr. Lee F. Ricketson from University of California, Los Angeles. The authors would like to thank them for these valuable discussions.

\section{References}

[1] Al-Mohssen, H.A. and Hadjiconstantinou, N.G. Low-variance direct Monte Carlo simulations using importance weights. ESAIM: M2AN, 44(5):1069-1083, 2010.

[2] L.L. Baker and N.G. Hadjiconstantinou. Variance reduction for Monte Carlo solutions of the Boltzmann equation. Physics of Fluids, 17:1-4, 2005.

[3] L.L. Baker and N.G. Hadjiconstantinou. Variance-reduced particle methods for solving the Boltzmann equation. J. Comput. Theor. Nanosci., 5:165-174, 2008.

[4] G.A. Bird. Molecular Gas Dynamics and the Direct Simulation of Gas Flows. Oxford University Press, London, 1998.

[5] A.V. Bobylev and K. Nanbu. Theory of collision algorithms for gases and plasmas based on the Boltzmann equation and the Landau-Fokker-Planck equation. Phys. Rev. E, 61:4576-4586, 2000 .

[6] R.E. Caflisch, C. Wang, G. Dimarco, B. Cohen, and A. Dimits. A hybrid method for accelerated simulation of Coulomb collisions in a plasma. Multiscale Modeling and Simulation, 7(2):865$887,2008$. 
[7] C. Cercignani. The Boltzmann equation and its applications. Springer-Verlag, 1988.

[8] J. Chun and D. L. Koch. A direct simulation Monte Carlo method for rarefied gas flows in the limit of small Mach number. Physics of Fluids (1994-present), 17(10):-, 2005.

[9] G. Dimarco, R.E. Caflisch, and L. Pareschi. Direct simulation Monte Carlo schemes for Coulomb interactions in plasmas. Commun. Appl. Indust. Math., 1:72-91, 2010.

[10] A.M. Dimits, C. Wang, R.E. Caflisch, B.I. Cohen, and Y. Huang. Understanding the accuracy of Nanbu's numerical Coulomb collision operator. J. Comput. Phys., 228(13):4881 - 4892, 2009.

[11] T.M. Homolle and N.G. Hadjiconstantinou. Low-variance deviational simulation Monte Carlo. Physics of Fluids, 19(4):-, 2007.

[12] T.M. Homolle and N.G. Hadjiconstantinou. A low-variance deviational simulation Monte Carlo for the Boltzmann equation. J. Comput. Phys., 226(2):2341-2358, October 2007.

[13] R.S. Martin and Jean-Luc Cambier. Moment preserving adaptive particle weights using octree velocity distributions for PIC simulations. AIP Conference Proceedings, 1501(1):872-879, 2012.

[14] K. Nanbu. Theory of cumulative small-angle collisions in plasmas. Phys. Rev. E, 55:4642-4652, 1997.

[15] L. Pareschi and G. Russo. An introduction to Monte Carlo method for the Boltzmann equation. ESAIM: Proc., 10:35-75, 2001.

[16] J-P.M Peraud, C.D. Landon, and N.G. Hadjiconstantinou. Monte carlo methods for solving the boltzmann transport equation. Annual Review of Heat Transfer, 17:205-265, 2014.

[17] L.F. Ricketson, M.S. Rosin, R.E. Caflisch, and A.M. Dimits. An entropy based thermalization scheme for hybrid simulations of Coulomb collisions. J. Comput. Phys., to appear, 2014.

[18] M.N. Rosenbluth, W.M. MacDonald, and D.L. Judd. Fokker-Planck equation for an inversesquare force. Phys. Rev., 107:1-6, Jul 1957.

[19] M. El Safadi. Smoothness of weak solutions of the spatially homogeneous Landau equation. Analysis and Applications, 05(01):29-49, 2007.

[20] M. Sherlock. A Monte-Carlo method for Coulomb collisions in hybrid plasma models. $J$. Comput. Phys., 227(4):2286 - 2292, 2008.

[21] T. Takizuka and H. Abe. A binary collision model for plasma simulation with a particle code. J. Comp. Phys., 25:205-219, 1977.

[22] C. Wang, T. Lin, R.E. Caflisch, B.I. Cohen, and A.M. Dimits. Particle simulation of Coulomb collisions: Comparing the methods of Takizuka \& Abe and Nanbu. J. Comput. Phys., 227(9):4308 - 4329, 2008.

\section{A Proof of Proposition 2.1}

The first equality (2.9) comes from the fact that, for any $\mathbf{u}$

$$
\int_{\mathbb{S}^{2}} D\left(\frac{\mathbf{u} \cdot \mathbf{n}}{u}, A \frac{\Delta t}{u^{3}}\right) \mathrm{d} \mathbf{n}=1
$$


Therefore

$$
\begin{aligned}
\int_{\mathbb{R}^{3}} P(\hat{g}, \hat{f}) \mathrm{d} \mathbf{v} & =\iiint_{\mathbb{R}^{3} \times \mathbb{R}^{3} \times \mathbb{S}^{2}} D\left(\frac{\mathbf{u} \cdot \mathbf{n}}{u}, A \frac{\Delta t}{u^{3}}\right) \hat{g}\left(\mathbf{w}^{\prime}, t\right) \hat{f}\left(\mathbf{v}^{\prime}, t\right) \mathrm{d} \mathbf{w} \mathrm{d} \mathbf{v} \mathrm{d} \mathbf{n} \\
& =\iiint_{\mathbb{R}^{3} \times \mathbb{R}^{3} \times \mathbb{S}^{2}} D\left(\frac{\mathbf{u} \cdot \mathbf{n}}{u}, A \frac{\Delta t}{u^{3}}\right) \hat{g}(\mathbf{w}, t) \hat{f}(\mathbf{v}, t) \mathrm{d} \mathbf{w} \mathrm{d} \mathbf{v} \mathrm{d} \mathbf{n} \\
& =\iint_{\mathbb{R}^{3} \times \mathbb{S}^{2}} \hat{g}(\mathbf{w}, t) \hat{f}(\mathbf{v}, t) \mathrm{d} \mathbf{w} \mathrm{d} \mathbf{v} \\
& =1
\end{aligned}
$$

where $\mathbf{u}=\mathbf{v}-\mathbf{w}$ and we have used the usual variable change $\mathbf{v}, \mathbf{w} \rightarrow \mathbf{v}^{\prime}, \mathbf{w}^{\prime}$, which leads to $\mathrm{d} \mathbf{v} \mathrm{d} \mathbf{w}=\mathrm{d} \mathbf{v}^{\prime} \mathrm{d} \mathbf{w}^{\prime}$ and $(\mathbf{v}-\mathbf{w}) \cdot \mathbf{n} \mathrm{d} \mathbf{n}=\left(\mathbf{v}^{\prime}-\mathbf{w}^{\prime}\right) \cdot \mathbf{n} \mathrm{d} \mathbf{n}$.

The second equality (2.10) also holds:

$$
\begin{aligned}
P(\hat{m}, \hat{m}) & =\iint_{\mathbb{R}^{3} \times \mathbb{S}^{2}} D\left(\frac{\mathbf{u} \cdot \mathbf{n}}{u}, A \frac{\Delta t}{u^{3}}\right) \hat{m}\left(\mathbf{w}^{\prime}, t\right) \hat{m}\left(\mathbf{v}^{\prime}, t\right) \mathrm{d} \mathbf{w} \mathrm{d} \mathbf{n} \\
& =\iint_{\mathbb{R}^{3} \times \mathbb{S}^{2}} D\left(\frac{\mathbf{u} \cdot \mathbf{n}}{u}, A \frac{\Delta t}{u^{3}}\right) \hat{m}(\mathbf{w}, t) \hat{m}(\mathbf{v}, t) \mathrm{d} \mathbf{w} \mathrm{d} \mathbf{n} \\
& =\hat{m}(\mathbf{v}) \int_{\mathbb{R}^{3}} \hat{m}(\mathbf{w}, t) \mathrm{d} \mathbf{w} \\
& =\hat{m}(\mathbf{v}),
\end{aligned}
$$

due to the fact that $m(\mathbf{v}) m(\mathbf{w})=m\left(\mathbf{v}^{\prime}\right) m\left(\mathbf{w}^{\prime}\right)$.

\section{B Some examples for $\tilde{D}$ in (4.5)}

The $\tilde{D}(\mu, \tau)$ term in (4.5) gives the distribution of the angle change $\theta=\arccos \mu$ in a collision with a parameter $\tau$. In this appendix we summarize these distributions for the widely used TA method and Nanbu method.

The collision model of the TA method ([6]) corresponds to

$$
\tilde{D}_{T A}(\mu, \tau)=\frac{1}{2 \pi} \frac{1}{\sqrt{2 \pi \tau}} e^{-\zeta^{2} / 2 \tau} \frac{\mathrm{d} \zeta}{\mathrm{d} \mu},
$$

with $\mu=\cos (2 \arctan \zeta)$.

The collision model of Nanbu's method ([5]) corresponds to

$$
\tilde{D}_{N a n b u}(\mu, \tau)=\frac{A}{4 \pi \sinh A} e^{\mu A},
$$

where $A$ is defined by

$$
\operatorname{coth} A-\frac{1}{A}=e^{-2 \tau}
$$

\section{Proof of Proposition 4.1}

In this appendix we give a proof of Proposition 4.1: a simplification of the formula of $\delta m\left(\mathbf{v} ; \mathbf{v}_{1}\right)$ and an approximation by a one dimensional integral. 


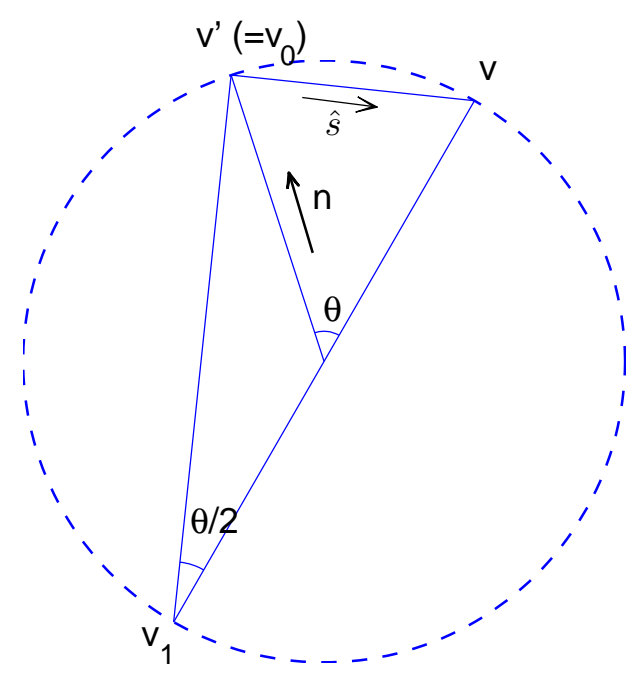

Figure 12: Illustration of the variables in Section C.1.

\section{C.1 Simplify $\delta m\left(\mathbf{v} ; \mathbf{v}_{1}\right)$}

Consider a source particle with fixed velocity $\mathbf{v}_{1}$ and an incident particle with velocity $\mathbf{v}$. After the collision of these two particles with collision angle $\mathbf{n} \in \mathbb{S}^{2}, \mathbf{v}$ changes to a new velocity

$$
\mathbf{v}^{\prime}=\frac{1}{2}\left(\mathbf{v}+\mathbf{v}_{1}\right)+\frac{1}{2}\left|\mathbf{v}-\mathbf{v}_{1}\right| \mathbf{n},
$$

as illustrated in Figure 12. Denote $\theta$ the angle between $\mathbf{n}$ and $\mathbf{v}-\mathbf{v}_{1}$, and

$$
\mu \doteq \cos \theta=\frac{\left(\mathbf{v}-\mathbf{v}_{1}\right) \cdot \mathbf{n}}{\left|\mathbf{v}-\mathbf{v}_{1}\right|}, \quad \tau=\tau(\mathbf{v}) \doteq A \Delta t\left|\mathbf{v}-\mathbf{v}_{1}\right|^{-3} .
$$

Let $D \doteq D\left(\frac{(\mathbf{v}-\mathbf{w}) \cdot \mathbf{n}}{|\mathbf{v}-\mathbf{w}|}, A \frac{\Delta t}{|\mathbf{v}-\mathbf{w}|^{3}}\right)$, then

$$
\int_{\mathbb{R}^{3}} D \delta\left(\mathbf{w}-\mathbf{v}_{1}\right) \mathrm{d} \mathbf{w}=D(\mu, \tau)
$$

Let $\phi$ be an angle over $[0,2 \pi]$. We can rewrite the collision kernel as

$$
D(\mu, \tau) \mathrm{d} \mathbf{n}=\tilde{D}(\mu, \tau) \mathrm{d} \mu \mathrm{d} \phi .
$$

Here $\tilde{D}$ is the collision kernel to be used in (4.5).

Now we can reduce the dimension of the integral in $\delta m\left(\mathbf{v} ; \mathbf{v}_{1}\right)$ since a $\delta$ function is involved. Following Bobylev and Nanbu's work [5], (4.4) can be reformulated as

$$
\begin{aligned}
& \delta m\left(\mathbf{v}_{0} ; \mathbf{v}_{1}\right)=\int \mathbb{R}_{\mathbb{R}^{3}} \delta\left(\mathbf{v}-\mathbf{v}_{0}\right) \delta m\left(\mathbf{v} ; \mathbf{v}_{1}\right) \mathrm{d} \mathbf{v} \\
&=\iiint_{\mathbb{R}^{3} \times \mathbb{R}^{3} \times \mathbb{S}^{2}} D m\left(\mathbf{v}^{\prime}\right) \delta\left(\mathbf{w}^{\prime}-\mathbf{v}_{1}\right) \delta\left(\mathbf{v}-\mathbf{v}_{0}\right) \mathrm{d} \mathbf{v} \mathrm{d} \mathbf{w} \mathrm{d} \mathbf{n}-m\left(\mathbf{v}_{0}\right) \\
&=\iiint_{\mathbb{R}^{3} \times \mathbb{R}^{3} \times \mathbb{S}^{2}} D m(\mathbf{v}) \delta\left(\mathbf{w}-\mathbf{v}_{1}\right) \delta\left(\mathbf{v}^{\prime}-\mathbf{v}_{0}\right) \mathrm{d} \mathbf{v} \mathrm{d} \mathbf{w} \mathrm{d} \mathbf{n}-m\left(\mathbf{v}_{0}\right) \\
& \stackrel{\text { by }}{\stackrel{(C .1)}{=}} \iint_{\mathbb{R}^{3} \times \mathbb{S}^{2}} D(\mu, \tau) m(\mathbf{v}) \delta\left(\mathbf{v}^{\prime}-\mathbf{v}_{0}\right) \mathrm{d} \mathbf{v} \mathrm{d} \mathbf{n}-m\left(\mathbf{v}_{0}\right) \\
& \stackrel{\text { by }}{=} \iiint \int_{\mathbb{R}^{3} \times[-1,1] \times[0,2 \pi]} \tilde{D}(\mu, \tau) m(\mathbf{v}) \delta\left(\mathbf{v}^{\prime}-\mathbf{v}_{0}\right) \mathrm{d} \mathbf{v} \mathrm{d} \mu \mathrm{d} \phi-m\left(\mathbf{v}_{0}\right) .
\end{aligned}
$$


We have used the usual variable changes $(\mathbf{v}, \mathbf{w}) \rightarrow\left(\mathbf{v}^{\prime}, \mathbf{w}^{\prime}\right)$ in the third line of (C.3). This variable change leads to $\mathrm{d} \mathbf{v} \mathrm{d} \mathbf{w}=\mathrm{d} \mathbf{v}^{\prime} \mathrm{d} \mathbf{w}^{\prime}$ and $(\mathbf{v}-\mathbf{w}) \cdot \mathbf{n} \mathrm{d} \mathbf{n}=\left(\mathbf{v}^{\prime}-\mathbf{w}^{\prime}\right) \cdot \mathbf{n} \mathrm{d} \mathbf{n}$.

Now we apply another variable change from $\mathbf{v}$ to $\mathbf{v}^{\prime}$. As illustrated in Figure 12, we have

$$
\mathbf{v}^{\prime}=\mathbf{v}_{1}+\cos (\theta / 2) R_{\theta / 2, \phi}\left(\mathbf{v}-\mathbf{v}_{1}\right),
$$

where $R_{\theta / 2, \phi}$ is a rotation matrix with angle $(\theta / 2, \phi)$. Therefore

$$
\mathrm{d} \mathbf{v}^{\prime}=\operatorname{det}\left(\cos (\theta / 2) R_{\theta / 2, \phi}\right) \mathrm{d} \mathbf{v}=\cos ^{3}(\theta / 2) \mathrm{d} \mathbf{v}=\left(\frac{1+\mu}{2}\right)^{3 / 2} \mathrm{~d} \mathbf{v}
$$

Then (C.3) gives

$$
\begin{aligned}
\delta m\left(\mathbf{v}_{0} ; \mathbf{v}_{1}\right) & =\iiint_{\mathbb{R}^{3} \times[-1,1] \times[0,2 \pi]}\left(\frac{1+\mu}{2}\right)^{-3 / 2} \tilde{D}(\mu, \tau) m(\mathbf{v}) \delta\left(\mathbf{v}^{\prime}-\mathbf{v}_{0}\right) \mathrm{d} \mathbf{v}^{\prime} \mathrm{d} \mu \mathrm{d} \phi-m\left(\mathbf{v}_{0}\right) \\
& =\iint_{[-1,1] \times[0,2 \pi]}\left(\frac{1+\mu}{2}\right)^{-3 / 2} \tilde{D}(\mu, \tau) m(\mathbf{v}) \mathrm{d} \mu \mathrm{d} \phi-m\left(\mathbf{v}_{0}\right),
\end{aligned}
$$

which means $\mathbf{v}^{\prime}$ is set to be $\mathbf{v}_{0}$ in Figure 12. Now for a fixed $\mathbf{v}_{1}$ and $\mathbf{v}_{0}$, we can express $\mathbf{v}$ as a function of the angles $\theta$ and $\phi$,

$$
\begin{aligned}
\mathbf{v}=\mathbf{v}(\mu, \phi) & =\mathbf{v}_{0}+\left|\mathbf{v}-\mathbf{v}_{0}\right| \hat{\mathbf{s}} \\
& =\mathbf{v}_{0}+\left|\mathbf{v}_{0}-\mathbf{v}_{1}\right| \tan (\theta / 2)\left(\hat{\mathbf{s}}_{1} \cos \phi+\hat{\mathbf{s}}_{2} \sin \phi\right) \\
& =\mathbf{v}_{0}+\left|\mathbf{v}_{0}-\mathbf{v}_{1}\right| \sqrt{\frac{1-\mu}{1+\mu}}\left(\hat{\mathbf{s}}_{1} \cos \phi+\hat{\mathbf{s}}_{2} \sin \phi\right),
\end{aligned}
$$

where $\hat{\mathbf{s}}=\left(\hat{\mathbf{s}}_{1} \cos \phi+\hat{\mathbf{s}}_{2} \sin \phi\right)$ is a unit vector in the plane perpendicular to $\left(\mathbf{v}_{0}-\mathbf{v}_{1}\right) ; \hat{\mathbf{s}}_{1}$ and $\hat{\mathbf{s}}_{2}$ are two orthogonal unit vectors in that plane. This gives the formula (4.6).

In addition, noting that

$$
\left|\mathbf{v}-\mathbf{v}_{1}\right|=\left|\mathbf{v}_{0}-\mathbf{v}_{1}\right| / \cos (\theta / 2)=((1+\mu) / 2)^{-1 / 2}\left|\mathbf{v}_{0}-\mathbf{v}_{1}\right|,
$$

one has

$$
\tau=A \Delta t\left|\mathbf{v}-\mathbf{v}_{1}\right|^{-3}=A \Delta t((1+\mu) / 2)^{3 / 2}\left|\mathbf{v}_{0}-\mathbf{v}_{1}\right|^{-3} \doteq \tau(\mu),
$$

which gives (4.7). Hence

$$
\begin{aligned}
\delta m\left(\mathbf{v}_{0} ; \mathbf{v}_{1}\right) & =\iint_{[-1,1] \times[0,2 \pi]}\left(\frac{1+\mu}{2}\right)^{-3 / 2} \tilde{D}(\mu, \tau(\mu)) m(\mathbf{v}(\mu, \phi)) \mathrm{d} \mu \mathrm{d} \phi-m\left(\mathbf{v}_{0}\right), \\
& =\int_{-1}^{1}\left(\frac{1+\mu}{2}\right)^{-3 / 2} 2 \pi \tilde{D}(\mu, \tau(\mu))\left(\frac{1}{2 \pi} \int_{0}^{2 \pi} m(\mathbf{v}(\mu, \phi)) \mathrm{d} \phi\right) \mathrm{d} \mu-m\left(\mathbf{v}_{0}\right),
\end{aligned}
$$

which gives (4.5).

\section{C.2 An approximation of $\delta m$}

One can further reduce the evaluation of $\Delta m$ in (4.5) to a one dimensional integral. We first give a lemma.

Lemma C.1. For a given unit vector $\mathbf{n}$ and a small number $\varepsilon$, we have the formula

$$
\frac{1}{2 \pi} \int_{\mathbf{s} \cdot \mathbf{n}=0, \| \mathbf{s}||=1} e^{-|\mathbf{v}-\varepsilon \mathbf{s}|^{2}} \mathrm{~d} \mathbf{s}=e^{-|\mathbf{v}|^{2}-\varepsilon^{2}} \sum_{k=0}^{\infty} \frac{1}{(k !)^{2}}\left(\varepsilon v_{\perp}\right)^{2 k},
$$


where

$$
\mathbf{v}_{\perp}=\mathbf{v}-(\mathbf{v} \cdot \mathbf{n}) \mathbf{n}, \quad v_{\perp}=\left|\mathbf{v}_{\perp}\right| .
$$

In particular, the cutoff after the third term gives

$$
\frac{1}{2 \pi} \int_{\mathbf{s} \cdot \mathbf{n}=0,\|\mathbf{s}\|=1} e^{-|\mathbf{v}-\varepsilon \mathbf{s}|^{2}} \mathrm{~d} \mathbf{s}=e^{-|\mathbf{v}|^{2}-\varepsilon^{2}}\left(1+\left(\varepsilon v_{\perp}\right)^{2}+\frac{1}{4}\left(\varepsilon v_{\perp}\right)^{4}\right)+\mathcal{O}\left(\varepsilon^{6}\right) .
$$

In addition,

$$
\frac{1}{2 \pi} \int_{\mathbf{s} \cdot \mathbf{n}=0, \| \mathbf{s}||=1} e^{-|\mathbf{v}-\varepsilon \mathbf{s}|^{2}} \mathrm{~d} \mathbf{s} \geq e^{-|\mathbf{v}|^{2}-\varepsilon^{2}}
$$

with equal sign holds if and only if $\mathbf{n}$ is parallel to $\mathbf{v}$.

The proof is straight forward.

For the Maxwellian

$$
m(\mathbf{v})=\frac{\rho_{m}}{\left(2 \pi T_{m}\right)^{3 / 2}} \exp \left(-\frac{\left|\mathbf{v}-\mathbf{u}_{m}\right|^{2}}{2 T_{m}}\right),
$$

and $\mathbf{v}(\mu, \phi)$ in (4.6), Lemma C.1 implies that in (4.5),

$$
\frac{1}{2 \pi} \int_{0}^{2 \pi} m(\mathbf{v}(\mu, \phi)) \mathrm{d} \phi=m\left(\mathbf{v}_{0}\right) e^{-\varepsilon^{2}}\left(1+\left(\varepsilon v_{\perp}\right)^{2}+\frac{1}{4}\left(\varepsilon v_{\perp}\right)^{4}+\mathcal{O}\left(\varepsilon^{6}\right)\right),
$$

where $\varepsilon$ and $v_{\perp}$ are given by (4.9) and (4.10). It follows that the integration (4.5) is approximated by the one dimensional integral in (4.8).

For TA method, plug in (B.1) and (4.7), and use substitution $\mu=\cos (2 \arctan \zeta)$, straightforward computation leads to (4.11).

\section{The upper bounds of $\delta m\left(\mathbf{v} ; \mathbf{v}_{1}\right)$ and $\Delta m(\mathbf{v})$}

In this appendix we study how to find upper bounds of $\delta m\left(\mathbf{v} ; \mathbf{v}_{1}\right)$ and $\Delta m(\mathbf{v})$. First we give a lemma on $\delta m\left(\mathbf{v}_{0} ; \mathbf{v}_{1}\right)$.

Lemma D.1 (The lower bound and upper bound of $\delta m\left(\mathbf{v} ; \mathbf{v}_{1}\right)$ ).

1. $\delta m\left(\mathbf{v} ; \mathbf{v}_{1}\right)$ is bounded from below. More specifically, with the positive constant $\alpha_{l}=\alpha_{l}\left(\Delta t, T_{m}\right)$ defined in (4.13), which only depends on time step length and the temperature of the Maxwellian part, one has $\alpha_{l}=\mathcal{O}(\Delta t)$ and

$$
\delta m\left(\mathbf{v} ; \mathbf{v}_{1}\right) \geq-\alpha_{l} m(\mathbf{v}), \quad \text { for any } \mathbf{v}, \mathbf{v}_{1} .
$$

2. For the TA method, $\delta m\left(\mathbf{v} ; \mathbf{v}_{1}\right)$ is singular around $\mathbf{v}=\mathbf{v}_{1}$,

$$
\delta m\left(\mathbf{v} ; \mathbf{v}_{1}\right)=\mathcal{O}\left(\left|\frac{\mathbf{v}-\mathbf{v}_{1}}{(\Delta t)^{1 / 3}}\right|^{-9 / 5}\right), \quad \text { as } \mathbf{v} \rightarrow \mathbf{v}_{1}
$$

Furthermore, for any $\beta>0$, with the positive constant $\alpha_{u}=\alpha_{u}\left(\Delta t, T_{m}, \beta\right)$ defined in (4.14), one has for any $\mathbf{v} \in \Omega_{\beta}:=\left\{\mathbf{v} \mid \frac{\left|\mathbf{v}-\mathbf{u}_{m}\right|}{\sqrt{2 T_{m}}}<\beta\right\}$,

$$
\left|\mathbf{v}-\mathbf{v}_{1}\right|^{2} \delta m\left(\mathbf{v} ; \mathbf{v}_{1}\right) \leq \alpha_{u} m(\mathbf{v}), \quad \text { for any } \mathbf{v}_{1} .
$$


Proof. First we study the lower bound. From (4.5) and (C.4), one has

$$
\delta m\left(\mathbf{v}_{0} ; \mathbf{v}_{1}\right) \geq m\left(\mathbf{v}_{0}\right) g_{l}\left(\Delta t, T_{m}, u_{01}\right),
$$

where

$$
g_{l}\left(\Delta t, T_{m}, u_{01}\right)=\int_{-1}^{1}\left(\frac{1+\mu}{2}\right)^{-3 / 2} 2 \pi \tilde{D}(\mu, \tau) e^{-\varepsilon^{2}} \mathrm{~d} \mu-1
$$

only depends on $\Delta t, T_{m}$, and the length of the relative velocity $u_{01}=\left|\mathbf{v}_{0}-\mathbf{v}_{1}\right|$. The equality sign in (D.1) is achieved when $\left(\mathbf{v}_{0}-\mathbf{v}_{1}\right)$ is parallel to $\left(\mathbf{v}_{0}-\mathbf{u}_{m}\right)$. Let

$$
-\alpha_{l}=\min _{u_{01}} g_{l}\left(\Delta t, T_{m}, u_{01}\right)=\min _{u_{01}} \int_{-1}^{1}\left(\frac{1+\mu}{2}\right)^{-3 / 2} 2 \pi \tilde{D}(\mu, \tau) e^{-\varepsilon^{2}} \mathrm{~d} \mu-1,
$$

then

$$
\delta m\left(\mathbf{v} ; \mathbf{v}_{1}\right) \geq-\alpha_{l} m(\mathbf{v}) .
$$

Next we investigate the upper bound. Let $u_{01}=\left|\mathbf{v}_{0}-\mathbf{v}_{1}\right| \rightarrow 0$, then $\varepsilon \rightarrow 0$ from (4.9). (4.5) and (4.8) leads to

$$
\delta m\left(\mathbf{v}_{0} ; \mathbf{v}_{1}\right) \approx m\left(\mathbf{v}_{0}\right) g_{u}\left(\Delta t, T_{m}, u_{01}\right),
$$

where

$$
g_{u}\left(\Delta t, T_{m}, u_{01}\right)=\int_{-1}^{1}\left(\frac{1+\mu}{2}\right)^{-3 / 2} 2 \pi \tilde{D}(\mu, \tau) \mathrm{d} \mu-1 .
$$

Plug in (B.1) and (4.7), and use substitution $\mu=\cos (2 \arctan \zeta)$, one has

$$
g_{u}\left(\Delta t, T_{m}, u_{01}\right)=\frac{r^{1 / 2}}{\sqrt{\pi}} \int_{0}^{\infty}\left(1+\zeta^{2}\right)^{9 / 4} e^{-r \zeta^{2}\left(1+\zeta^{2}\right)^{3 / 2}} \mathrm{~d} \zeta-1,
$$

with $r=\frac{\left|\mathbf{v}_{0}-\mathbf{v}_{1}\right|^{3}}{2 A \Delta t}$.

Note that

$$
\begin{aligned}
\int_{0}^{\infty}\left(1+\zeta^{2}\right)^{9 / 4} e^{-r \zeta^{2}\left(1+\zeta^{2}\right)^{3 / 2}} \mathrm{~d} \zeta & \leq \int_{0}^{1} 2^{9 / 4} \mathrm{~d} \zeta+2^{9 / 4} \int_{1}^{\infty} \zeta^{9 / 2} e^{-r \zeta^{5}} \mathrm{~d} \zeta \\
& =2^{9 / 4}+2^{9 / 4} r^{-11 / 10} \int_{r^{1 / 5}}^{\infty} x^{9 / 2} e^{-x^{5}} \mathrm{~d} x \\
& =\mathcal{O}\left(r^{-11 / 10}\right), \quad \text { as } r \rightarrow 0,
\end{aligned}
$$

one has

$$
g_{u}\left(\Delta t, T_{m}, u_{01}\right) \rightarrow \mathcal{O}\left(r^{-3 / 5}\right)=\mathcal{O}\left(\left|\frac{\mathbf{v}_{0}-\mathbf{v}_{1}}{(\Delta t)^{1 / 3}}\right|^{-9 / 5}\right) .
$$

Finally, we define constants $c_{0}, c_{1}$ and $c_{2}$ as in (4.15). Note that from (4.10)

$$
v_{\perp} \leq \frac{1}{\sqrt{2 T_{m}}}\left|\mathbf{v}_{0}-\mathbf{u}_{m}\right| \leq \beta,
$$

in the region $\mathbf{v}_{0} \in \Omega_{\beta}$. Take

$$
\alpha_{u}=\alpha_{u}(\beta)=c_{0}+\beta^{2} c_{1}+\beta^{4} c_{2},
$$

then for $\mathbf{v} \in \Omega_{\beta}$

$$
\left|\mathbf{v}-\mathbf{v}_{1}\right|^{2} \delta m\left(\mathbf{v} ; \mathbf{v}_{1}\right) \leq \alpha_{u} m(\mathbf{v})
$$


This lemma leads to the first part in Proposition 4.4. For the remaining part, one has

$$
\begin{aligned}
\left|\Delta m_{+}(\mathbf{v})\right| & \leq \frac{N_{\text {eff }}}{\rho} \sum_{\mathbf{v}_{p}} \delta m_{+}\left(\mathbf{v} ; \mathbf{v}_{p}\right)+\frac{N_{\text {eff }}}{\rho} \sum_{\mathbf{v}_{n}} \delta m_{+}\left(\mathbf{v} ; \mathbf{v}_{n}\right) \\
& \leq \frac{\alpha_{u} N_{\text {eff }}}{\rho} m(\mathbf{v})\left(\sum_{\mathbf{v}_{p}} \frac{1}{\left|\mathbf{v}-\mathbf{v}_{p}\right|^{2}}+\sum_{\mathbf{v}_{n}} \frac{1}{\left|\mathbf{v}-\mathbf{v}_{n}\right|^{2}}\right) \\
& \leq \frac{\alpha_{u} N_{\mathrm{eff}}}{\rho} \frac{\rho_{m}}{\left(2 \pi T_{m}\right)^{3 / 2}}\left(\sum_{\mathbf{v}_{p}} \frac{1}{\left|\mathbf{v}-\mathbf{v}_{p}\right|^{2}}+\sum_{\mathbf{v}_{n}} \frac{1}{\left|\mathbf{v}-\mathbf{v}_{n}\right|^{2}}\right)=\overline{\Delta m_{+}}(\mathbf{v}), \\
\left|\Delta m_{-}(\mathbf{v})\right| & \leq \frac{N_{\mathrm{eff}}}{\rho} \sum_{\mathbf{v}_{p}} \delta m_{-}\left(\mathbf{v} ; \mathbf{v}_{p}\right)+\frac{N_{\mathrm{eff}}}{\rho} \sum_{\mathbf{v}_{n}} \delta m_{-}\left(\mathbf{v} ; \mathbf{v}_{n}\right) \\
& \leq \frac{\alpha_{l} N_{\mathrm{eff}}}{\rho}\left(N_{p}+N_{n}\right) m(\mathbf{v})=\overline{\Delta m_{-}}(\mathbf{v}) .
\end{aligned}
$$

